\title{
LE PENTATEUQUE TOUJOURS EN QUESTION: BILAN ET PERSPECTIVES APRÈS UN QUART DE SIÈCLE DE DÉBAT
}

\author{
Thomas Römer
}

Lausanne

\section{o. Introduction: A la recherche d'un nouveau consensus}

Depuis un quart de siècle, les exégètes parlent d'une mise en question, voire d'une "crise" du Pentateuque. Bien entendu, cette crise ne concerne pas la Torah comme document fondateur du judaïsme, ou le Pentateuque en tant qu'il sert d'ouverture à la Bible chrétienne. Ce qui est en crise, c'est l'analyse historique du Pentateuque, autrement dit les modèles explicatifs qui ont été avancés pour la formation des cinq premiers livres de la Bible et l'identification de leurs contextes de production. A bien des égards, on peut même dire que la crise s'est aggravée durant ces dix dernières années, à tel point que l'absence de tout consensus n'exaspère pas seulement les étudiants mais aussi certains collègues qui cherchent désormais le salut dans les approches dites "synchroniques", narratologie ou autres holistic readings. Il n'y a aucun doute que certaines méthodes de l'exégèse historique devraient faire l'objet d'un examen critique (je pense notamment ici à une certaine Literarkritik allemande prétendant reconstruire au demi-verset près des documents anciens, nous y reviendrons). Toutefois, il ne saurait être sérieusement question de renoncer à l'approche historique de la Bible hébraïque en général, et du Pentateuque en particulier, au seul prétexte du caractère hypothétique des résultats obtenus.

Le grand bouleversement dans les recherches historico-critiques sur le Pentateuque peut être situé au milieu des années 1970 avec la parution des livres de John Van Seters, Hans Heinrich Schmid et Rolf Rendtorff.' Ces auteurs, dont il n'est plus nécessaire de résumer les

' J. Van Seters, Abraham in History and Tradition (New Haven-London, 1975); H.H. Schmid, Der sogenannte Fahwist. Beobachtungen und Fragen zur Pentateuchforschung (Zürich, 1976); R. Rendtorff, Das überlieferungsgeschichtliche Problem des Pentateuch, BZAW 147 (Berlin, New York, 1976). 
positions, ${ }^{2}$ mirent chacun à leur manière le doigt sur les faiblesses de la théorie documentaire, mais aussi de certaines conceptions historiques sur lesquelles cette théorie reposait. Bien que ces nouveaux modèles aient d'abord fait l'objet de violentes réfutations, on vit apparaitre vers la fin des années quatre-vingt de nouvelles possibilités de consensus. Les travaux d'Erhard Blum, qui proposait de voir dans le Pentateuque la résultante de la combinaison entre une "composition D" (post-dtr) et une "composition P" (sacerdotale) étaient partiellement compatibles avec les conceptions du Yahwiste avancées par Van Seters et Martin Rose. Tout comme la composition D, ce Yahwiste présupposait l'historiographie dtr, mais précédait les textes d'origine sacerdotale. Dès lors, la plupart des chercheurs qui acceptaient la mise en question du paradigme de Julius Wellhausen--revu et corrigé par Martin Noth et Gerhard von Rad-partageaient certains points de repère, que l'on peut résumer à grands traits de la manière suivante.

Dans la perspective de ce nouveau consensus, le Pentateuque se composait avant tout d'un document laïc $(\mathrm{J}$ ou $\mathrm{D}),{ }^{3}$ datant au plus tôt de l'époque exilique mais intégrant vraisemblablement des données plus anciennes, et de textes sacerdotaux, qui soit auraient formé un document indépendant à l'origine, soit au contraire auraient servi à rééditer l'œuvre laïque. Nombreux furent ceux qui postulèrent également un lien étroit entre la publication du Pentateuque et les circonstances de la soi-disant "autorisation impériale", prétendument décrétée dans la province de Yehud par l'administration achéménide. La Torah apparaissait ainsi comme un document de compromis entre le clergé de Jérusalem et le pouvoir "laique". Plusieurs auteurs avaient ainsi l'impression que certains piliers de la recherche sur le Pentateuque, peu ou pas contestés, permettaient d'envisager une nouvelle théorie d'ensemble sur la formation du Pentateuque. En particulier, la coexistence de deux documents, sacerdotal et non sacerdotal, couvrant l'ensemble des cinq premiers livres de la Bible semblait assurée. L'existence d'une "histoire deutéronomiste", dont le Deutéronome aurait constitué l'ouverture à l'origine, semblait également inébranlable—ce qui explique au passage

${ }^{2}$ Cf. la présentation dans A. de Pury et T. Römer, "Le Pentateuque en question: position du problème et brève histoire de la recherche," dans A. de Pury (éd.), Le Pentateuque en question, Le Monde de la Bible 19 (Genève, ${ }^{2}$ I99I), pp. 9-80, en particulier pp. $57-66$.

${ }^{3}$ Le document "élohiste" avait disparu du paysage exégétique ou était devenu une couche rédactionnelle aux contours peu clairs; cf. par contre H. Seebass, "Pentateuch," TRE 26 (1996), pp. I85-209. 
que le dernier livre du Pentateuque ait finalement été peu présent dans le débat sur la formation des cinq premiers livres de l'AT. En outre, la théorie de l'autorisation impériale semblait offrir un contexte historique clair, sinon évident, pour expliquer la publication de la Torah.

Or, force est de constater que tous ces piliers sont aujourd'hui fortement ébranlés. Certes, personne ne nie l'existence de textes de type "sacerdotal" dans le Pentateuque, mais le débat sur la fin de la version primitive de ce document est agité, et il risque de bouleverser certaines idées sur P. Par ailleurs, la contestation de l'existence d'une histoire dtr gagne du terrain depuis une dizaine d'années. Et cette contestation s'accompagne du retour de la théorie de l'Hexateuque, théorie qui avait quasiment disparu de la discussion exégétique depuis Noth. L'hypothèse d'une oeuvre "yahwiste" ou d'une "composition D" qui auraient couvert le Tétrateuque, voire le Pentateuque, se trouve également mise à mal par des travaux récents qui soulignent l'indépendance stylistique et idéologique de certains des "grands ensembles" au sein de la Torah. Et, last but not least, la théorie de l'autorisation impériale semble également avoir du plomb dans l'aile.

L'objet de cette contribution n'est pas de fournir une histoire exhaustive de la recherche, ${ }^{4}$ mais de reprendre de manière un peu plus détaillée les développements récents qui viennent d'être esquissés.

${ }^{4}$ Cf. notamment les publications suivantes: F. García López, "De la antigua a la nueva crítica literaria del Pentateuco," Estudios Biblicos 52 (1994), pp. 7-35; C. Houtman, Der Pentateuch. Die Geschichte seiner Erforschung nebst einer Auswertung (Kampen, 1994); R. David, "Le Pentateuque. Tendances actuelles concernant les traditions littéraires," dans M. Gourgues et L. Laberge (éd.), "De bien des manières". La recherche biblique aux abords du XXème siècle, LD I63 (Montréal, Paris, I995); L. Schmidt, "Zur Entstehung des Pentateuch. Ein kritischer Literaturbericht"; VuF 4o (1995), pp. 3-28; T. Römer, "La formation du Pentateuque selon l'exégèse historico-critique," dans C.-B. Amphoux et J. Margain (éd.), Les premières traditions de la Bible (Prahins, 1996); J.-L. Ska, "Le Pentateuque: état de la recherche à partir de quelques récentes 'Introductions'," Bib 77 (1996), pp. 245-265; D.M. Carr, "Controversy and Convergence in Recent Studies of the Formation of the Pentateuch," Religious Studies Review 23 (I997), pp. 223I; R. Rendtorff, "Directions in Pentateuchal Studies," CR:BS 5 (1997), pp. 43-65; D.J. Wynn-Williams, The State of the Pentateuch: A Comparison of the Approaches of M. Noth and E. Blum, BZAW 249 (Berlin, New York, 1997); O. Artus, Le Pentateuque, CE Io6 (Paris, 1998); E. Nicholson, The Pentateuch in the Twentieth Century: The Legacy of Julius Wellhausen (Oxford, I998); G. Fischer, "Wege aus dem Nebel? Ein Beitrag zur Pentateuchkrise," BN 99 (1999), pp. 5-7; E. Otto, "Deuteronomium und Pentateuch. Aspekte der gegenwärtigen Debatte," ZAR 6 (2000), pp. 222-284; O. Kaiser, "The Pentateuch and the Deuteronomistic History," dans A.D.H. Mayes (éd.), Text in Context: Essays by Members of the Society for Old Testament Studies (Oxford, 2000). 


\section{Le débat sur "P"}

\section{I.I. Source ou rédaction?}

La mise en question de la théorie documentaire n'avait pas directement affecté les textes sacerdotaux, dont la délimitation pose relativement peu de problèmes. Pourtant, Van Seters et Rendtorff avaient considéré à la suite de Frank Moore Cross et d'autres ${ }^{5} \mathrm{P}$ comme une rédaction tardive dans le Pentateuque, qui aurait achevé ce dernier en complétant le matériel d'origine non sacerdotale. Cette position, qui renoue ainsi avec une sorte de théorie des compléments, est relativement répandue dans l'exégèse américaine, alors que l'exégèse germanophone continue dans sa grande majorité à distinguer entre une Grundschrift indépendante $\left(\mathrm{P}^{g}\right)$ à l'origine et plusieurs adjonctions successives $\left(\mathrm{P}^{\mathrm{s}}\right){ }^{6}$

Les tenants d'un $\mathrm{P}$ rédacteur font observer qu'il est impossible de reconstruire un document $P$ entièrement autonome et cohérent. Ainsi, en Ex 6,2, Moïse apparaît dans le récit sacerdotal sans avoir été mentionné auparavant. Néanmoins, il est indubitable à mon sens que certaines observations s'expliquent mieux dans le cadre de l'hypothèse d'un document $\mathrm{P}$ indépendant à l'origine. On peut penser notamment au fait qu'Ex 6,2 fournit une bonne suite au texte $\mathrm{P}$ d'Ex 2,23aß25. De même, il est difficile d'interpréter la version sacerdotale d'Ex I4 comme une simple rédaction de la version antérieure; et on peut aisément reconstruire un récit sacerdotal en Ex $7-12^{*}$ qui ne parle pas de "plaies" mais des manifestations de la puissance de Yhwh. En bref, il semble possible d'extraire pour certaines parties du Pentateuque (notamment pour $\mathrm{Ex} \mathrm{I}^{-} \mathrm{I}$ ) un récit $\mathrm{P}$ indépendant et cohérent, bien qu'il faille admettre quelques lacunes (ainsi par ex. dans l'histoire de Jacob, où $\mathbf{P}$ ne contient pas de mention des mariages de Jacob et de ses enfants). D'un autre côté, certains textes de facture sacerdotale ont clairement une fonction rédactionnelle et éditoriale, notamment les textes soi-disant sacerdotaux dans le livre des Nombres (par ex. Nb I3-I4 et

${ }^{5}$ F.M. Cross, Canaanite Myth and Hebrew Epic: Essays in the History of the Religion of Israel (Cambridge MA, I973), pp. 293-325; cf. déjà par exemple R.H. Pfeiffer, Introduction to the Old Testament (New York, ${ }^{2}$ I948), pp. I88-209; P. Volz, "P ist kein Erzähler," dans P. Volz, W. Rudolph, Der Elohist als Erzähler. Ein Irrweg der Pentateuchkritik, BZAW 63 (Giessen, 1933), pp. I35-142.

${ }^{6}$ Cf. A. Campbell, "The Priestly Text: Redaction or Source?," dans G. Braulik, W. Groß et S. McEvenue (éd.), Biblische Theologie und gesellschaftlicher Wandel. Festschrift. für Norbert Lohfink (Freiburg i.B.-Basel-Wien, 1993), pp. 32-47. 
$\left.16-17^{7}\right)$. Erhard Blum et Jean-Louis Ska ont parlé de "la relative indépendance de l'écrit sacerdotal". ${ }^{8}$ A mon sens, cette observation s'explique par le fait qu'il faut distinguer entre un document qui était originellement destiné à être publié et lu tel quel ( $\left.\mathrm{P}^{\mathrm{g}}\right)$, et une ou plusieurs rédactions de type sacerdotal, dont le profil reste à définir.

\subsection{Le problème de la fin de $P^{g}$}

Si nous conservons le terme de Grundschrift pour le document sacerdotal original, la question se pose du coup quant à l'étendue de cet écrit. Les bouleversements des années 1970 n'avaient guère affecté le problème de la fin du document sacerdotal, et la plupart des auteurs suivaient Noth qui considérait que $\mathrm{Pg}^{\mathrm{g}}$ se terminait avec la mort de Moïse en Dt 34, I $^{*} .7-9$. Cette option de Noth s'explique avant tout par son rejet d'un Hexateuque, autrement dit par le refus que les sources du Pentateuque aient pu se poursuivre en Josué, selon une hypothèse qui s'était imposée à la plupart des exégètes à la suite de Wellhausen. Mais Noth trouve également une justification théologique à cette finale en décrétant l'absence d'intérêt de $\mathrm{P}$ pour le pays. ${ }^{9}$ En réalité, on pourrait dire en exagérant quelque peu que le modèle de Noth ne lui laissait guère d'autre choix que Dt 34,7-9 pour la conclusion de Ps, et ceci pour deux raisons. D'une part, la thèse d'une histoire dtr était difficile à concilier avec l'existence d'un Hexateuque; d'autre part, P était pour Noth la "literarische Grundlage" du Pentateuque, ${ }^{10}$ et devait par conséquent nécessairement s'étendre de Gn I à Dt 34. Jusqu'en I988, la solution de Noth était majoritairement acceptée pour la fin de $\mathrm{P}$. La parution à cette date d'un article de Lothar Perlitt ${ }^{11}$ changea la donne. Perlitt y montrait en effet que Dt 34,7-9 pouvait difficilement être attribué à $\mathrm{Pg}$. Ces versets présupposent des textes comme $\mathrm{Nb}$ 27,12-23 qui sont

7 Contra L. Schmidt, Studien zur Priesterschrift, BZAW 2I4 (Berlin, New York, I993), pp. 73-179; cf. O. Artus, Etudes sur le livre des Nombres. Récit, Histoire et Loi en Nombres I3,I-20,I3, OBO ${ }_{157}$ (Fribourg CH, Göttingen, 1997), pp. 83-203; et surtout E. Otto, "Forschungen zur Priesterschrift," Theologische Rundschau 62 (1997), pp. I-50, ici pp. I6I8.

${ }^{8}$ Cf. Blum, Studien, pp. 221-228; J.-L. Ska, "De la relative indépendance de l'écrit sacerdotal," Bib 76 (1996), pp. 396-415.

${ }^{9}$ M. Noth, Überlieferunsgeschichte des Pentateuch (Stuttgart, 1948), p. 253.

${ }^{10} \mathrm{M}$. Noth, Überlieferungsgeschichtliche Studien. Die sammelnden und bearbeitenden Geschichtswerke im Alten Testament (1943) (Darmstadt, 1967), p. 206.

11 L. Perlitt, "Priesterschrift im Deuteronomium?" (1988), dans Deuteronomium-Studien, FAT 8 (Tübingen, 1994), pp. I23-I43. 
vraisemblablement secondaires. ${ }^{12}$ En outre, le vocabulaire de Dt $34,7-9$ témoigne d'un mélange de style sacerdotal et de style dtr, et provient par conséquent non pas de $\mathrm{Pg}$, mais d'une rédaction beaucoup plus tardive. Cette mise à mort de $\mathrm{P}^{\mathrm{g}}$ en $\mathrm{Dt} 34$ n'a toutefois pas convaincu tout le monde, puisque d'autres auteurs ont continué de défendre la solution de Noth.

\section{I.2.1. Deutéronome $34^{*}$}

C'est le cas notamment de Ludwig Schmidt dans ses Studien zur Priesterschrift. Pour Schmidt, le fait que le document sacerdotal se termine par la mort de Moïse et l'installation de Josué comme son successeur montre que $\mathrm{P}$, tout en connaissant l'histoire $\mathrm{dtr}$, veut instaurer avec la mort de Moïse une coupure qualitative. Seule l'époque mosaïque est vraiment fondatrice pour la communauté judéenne du deuxième temple. On peut néanmoins se demander si cette mise en évidence de la figure de Moïse comme principe structurant du Pentateuque est réellement le fait de $\mathrm{P}$, et ce d'autant plus qu'on n'attribue traditionnellement à $\mathrm{P}^{\mathrm{g}}$ que peu d'intérêt pour la figure de Moïse. En outre, on peut douter que Dt 34,9 (qui introduit Josué comme successeur de Moïse) constitue une fin idéale pour $\mathrm{Pg}$, alors que ce verset prépare clairement la suite du récit en Jos iss. Christian Frevel, ${ }^{13}$ qui défend également l'idée d'une fin de Ps en Dt 34, a d'ailleurs bien senti le problème inhérent à la solution traditionnelle et considère que la version primitive de $\mathrm{P}$ se serait terminée en 34,8 (avec la notice sur l'achèvement du deuil des Israélites), ce qui n'est guère une solution plus heureuse. Frevel reconnaît également que cette solution oblige à postuler la suppression du récit de la mort de Moïse proprement dit, à moins que celui-ci ne puisse être isolé au v. 5-un verset qui est toutefois plus dtr que sacerdotal, ${ }^{14}$. Le fait qu'un récit sacerdotal complet et cohérent ne puisse être reconstruit en Dt 34 devrait plutôt conduire à considé-

\footnotetext{
${ }^{12}$ Cf. également P. Stoellger, "Deuteronomium 34 ohne Priesterschrift," $Z A W$ I05 (I993), pp. 26-5I.

${ }_{13}$ C. Frevel, Mit Blick auf das Land die Schöpfung erinnern. Zum Ende der Priestergrundschrift, HBSt 23 (Freiburg et al., 1999).

14 Frevel, Blick, pp. 337-338. Le titre de "serviteur de Yhwh" est incontestablement dtr, et les raisons pour lesquelles l'expression 'על-פי יהוה' devrait être typique de $\mathrm{P}$ m'échappent. L'expression se rencontre en effet souvent en $\mathrm{Nb}$ iss., mais ígalement en ${ }_{2} \mathrm{R} 23,25$ et dans bien d'autres livres. Il s'agit donc d'un "frequent idiom" (A.D.H. Mayes, Deuteronomy, NCBC [Grand Rapids-London, I98I], p. 413).
} 
rer les v. 7-9 comme faisant partie d'une intervention rédactionnelle. Les arguments de Perlitt concernant l'absence de $\mathrm{P}^{\mathrm{g}}$ en Dt 34 restent toujours valables.

\subsubsection{Pg en fosué?}

Certains auteurs (Norbert Lohfink, Joseph Blenkinsopp, Ernst-Axel Knauf, Horst Seebass) reviennent alors à une idée déjà émise par Nöldeke et Wellhausen ${ }^{15}$ et trouvent la conclusion de $\mathrm{P}^{\mathrm{g}}$ dans la fin du livre de Josué, à savoir soit en Jos i8, I ("Toute la communauté des fils d'Israël s'assembla à Shilo et on y installa la tente de la rencontre. Le pays leur était soumis"; Seebass, Knauf), ${ }^{16}$ soit en I9,5I ("... Ils achevèrent ainsi le partage du pays"; Lohfink, Blenkinsopp). ${ }^{17}$ Indépendamment du fait que le fil du récit sacerdotal en Josué est franchement ténu et que la plupart des textes attribués à $\mathbf{P}$ dans ce livre sont en réalité plutôt des textes "mixtes" (de facture dtr et sacerdotale), la finale proposée ne semble guère convaincante. D'abord, on s'explique mal le soudain intérêt de $\mathrm{P}^{\mathrm{g}}$ pour Shilo. La soi-disant inclusion entre Gn I,28 et Jos I8, I (avec la reprise de la racine כבש n'existe pas, ${ }^{18}$ car Gn I,28 parle de la domination de la terre et des animaux par tous les humains. Ce projet échoue d'abord (Gn 6,II-I2), puis est corrigé et confirmé en Gn 9,I-7*. De plus, la structure de $\mathrm{P}$ en trois époques, caractérisées par la révélation des noms divins Elohim (origines), El Shadday (Patriarches) et Yhwh (Moïse), suggère plutôt que l'aboutissement de la version sacerdotale de base consistait dans la révélation de Yhwh à Israël.

15 Cf. à ce propos Frevel, Blick, pp. 9-15. Il faut signaler une certaine hésitation à ce propos chez Wellhausen. Dans sa Composition des Hexateuch (Berlin, ${ }^{4}$ I964), il semble opter pour Jos 22,34 (p. I28; cf. par contre p. 352), alors que dans les Prolegomena zur Geschichte Israels (Berlin, ${ }^{3} \mathrm{I} 886$ ), il envisage plutôt Dt 34 .

${ }^{16}$ Seebass, "Pentateuch," p. I92; cf. également Id., "Josua," BN 28 (I985), pp. 5365 , p. 58; E.A. Knauf, "Die Priesterschrift und die Geschichten der Deuteronomisten," dans T. Römer (éd.), The Future of the Deuteronomistic History, BETL I47 (Leuven, 2000), pp. II4-II5.

17 N. Lohfink, "Die Priesterschrift und die Geschichte," dans J. A. Emerton et al. (éd.), Congress Volume. Göttingen 1977, VT.S 29 (Leiden, 1978), pp. 189-225 = Studien zum Pentateuch, SBAB 4 (Stuttgart, 1988), pp. 213-253. Traduction française (partielle): "L'écrit sacerdotal et l'histoire," dans Les traditions du Pentateuque autour de l'exil, CE 97 (Paris, 1996), pp. 9-25; ici p. I4; J. Blenkinsopp, The Pentateuch. An Introduction to the First Five Books of the Bible, The Anchor Bible Reference Library (New York et al., I992), p. 237.

${ }_{18}$ On pourrait d'ailleurs aussi bien arguer d'une inclusion avec $\mathrm{Nb} 33,22.29$, mais ces textes sont généralement considérés comme "secondaires". 


\section{I.2.3. Ps en Nombres?}

Si Ps n'est présent ni en Dt ni en Jos, d'autres possibilités doivent être envisagées. Théoriquement, on pourrait chercher la fin de $\mathrm{P}$ quelque part en Nombres, ${ }^{19}$ mais aucun texte ne semble constituer un candidat satisfaisant. Il apparaît d'ailleurs de plus en plus difficile de trouver dans ce livre des textes qui puissent être attribués sans problème à $\mathrm{P}^{5} .{ }^{20}$ Ainsi, Thomas Pola a rendu plausible l'idée que $\mathrm{Nb}$ I-Io serait à considérer comme un ensemble d'ajouts tardifs composés dans le style sacerdotal. ${ }^{21}$ Il fait remarquer que la description du campement présente Israël comme une ecclesia militans, ce qui contredit le pacifisme qu'on se plaît souvent à souligner chez l'auteur sacerdotal. Erik Aurelius a observé que les récits de révoltes en $\mathrm{Nb}$ I3-I4; I6-I7 et 20 contredisent d'une certaine manière l'anthropologie de $\mathrm{P}^{\mathrm{g}}$, selon laquelle l'homme n'a de toute façon pas la possibilité de s'opposer aux projets du Dieu créateur. Au contraire, $\mathrm{P}$ insiste en général abondamment sur le fait que les fils d'Israël agirent exactement selon ce que Yhwh leur avait ordonné de faire. ${ }^{22}$

${ }^{19}$ Cf. les réflexions de J.-L. Ska, Introduction à la lecture du Pentateuque. Clés pour l'interprétation des cinq premiers lizres de la Bible, Le livre et le rouleau 5 (Bruxelles, 2000), pp. 210-214, qui envisage à la suite de Perlitt ("Priesterschrift") Nb 27. Cf. encore B. Janowski, "Tempel und Schöpfung. Schöpfungstheologische Aspekte der priesterschriftlichen Heiligtumskonzeption," JBTh 5 (1990), pp. 37-69 = Gottes Gegenwart in Israel. Beiträge zur Theologie des Alten Testaments (Neukirchen-Vluyn, I993), pp. 214-246, p. 224. Néanmoins, dans la mesure où ce texte soulève exactement les mêmes difficultés que Dt 34,7-9, il paraît également difficile d'y retrouver la finale de la version primitive de $\mathrm{P}$.

${ }^{20}$ O. Kaiser, Grundriß der Einleitung in die kanonischen und deuterokanonischen Schriften des Alten Testaments. Band I: Die erzählenden Werke (Gütersloh, I992), p. 58, stipulait que P "läßt sich mit Sicherheit bis Num 10,9 verfolgen" (il est néanmoins plus prudent dans Der Gott des Alten Testaments. Theologie des Alten Testaments. Teil I: Grundlegung, UTB I747 [Göttingen, I993], p. I8o, où il envisage $\mathrm{Nb}$ 20,22-29, tout en admettant la possibilité qu'il s'agisse là d'un texte "sekundärpriesterlich"). La question se pose toutefois de savoir si le départ du Sinaï constitue une fin concevable (cf. à ce sujet les remarques de Frevel, Blick, pp. $7 \mathrm{I}-72)$.

${ }_{21}$ T. Pola, Die ursprüngliche Priesterschrift. Beobachtungen zur Literarkritik und Traditionsgeschichte von Pg, WMANT 70 (Neukirchen-Vluyn, 1995), pp. 56-92. Pola se base surtout sur le travail de D. Kellermann, Die Priesterschrift von Num I,I bis Io,Io literarkritisch und traditionsgeschichtlich untersucht, BZAW I20 (Berlin, 197o).

22 E. Aurelius, Der Fürbitter Israels. Eine Studie zum Mosebild im Alten Testament, CB.OT 27 (Stockholm, I988), pp. 187-202. Cf. également Kaiser, Gott, pp. I80-182; Pola, Priesterschrift, pp. 92-99. 


\subsubsection{Pg dans la péricope du Sinä}

$\mathrm{Si}$ aucun texte de $\mathrm{Nb}$ n'offre une finale à $\mathrm{Pg}$, il faut envisager la possibilité d'une conclusion dans la péricope du Sinaï. Rappelons d'abord que Noth avait lui-même considéré la théophanie au Sinaï comme le "eigentliche Ziel" (le véritable but) de l'écrit sacerdotal. ${ }^{23}$ Pola s'est efforcé de montrer que cette observation de Noth devait également servir à délimiter l'étendue de $\mathrm{P}^{\mathrm{g}}$. Pola souligne d'abord que la fin du livre de l'Exode (la construction du sanctuaire et l'installation de Yhwh dans celui-ci) renvoie très clairement à $\mathrm{Gn}$ I, selon une constante dans la mythologie du Proche Orient ancien où la création s'achève fréquemment par l'érection d'un sanctuaire pour le Dieu créateur. Pola veut dater $\mathrm{P}^{\mathrm{g}}$ avant la construction du deuxième temple, et le considère largement influencé par la prédication d'Ezéchiel, notamment Ez 20. Outre les parallèles évidents entre $\mathrm{Ex} 6,2-8$ et $\mathrm{Ez}$ 20,5-9, il existe un autre point de concordance entre ces deux ensembles littéraires: selon Ez 20,40 le but de toute l'histoire de Yhwh avec Israël se trouverait dans le culte que son peuple lui rendrait au mont Sion. Pola en conclut que le sanctuaire qui est construit en finale du livre de l'Ex n'était pas, à l'origine, une demeure mobile mais la transposition du temple de Jérusalem dans le désert, le Sinaï devant alors être compris, dans cette perspective, comme un substitut ou un symbole pour Sion. Il s'ensuit que tous les textes qui insistent sur la mobilité du sanctuaire sont à considérer comme secondaires. Selon Pola, la fin du récit de $\mathrm{Pr}$ au Sinaï comporte seulement les versets suivants: Ex I9, I; 24,I5b-I8a; 25, I.8a. 9; 29,45s.; 40,16-17a. 33b. Cette solution soulève néanmoins certaines difficultés. L'idée d'une finale de $\mathrm{Pg}^{\mathrm{g}}$ en Ex 40 est certainement séduisante, ${ }^{24}$ mais peut-on se contenter d'extraire environ $2 \%$ du texte actuel d'Ex 25-40, et considérer tout le reste comme secondaire? Peut-on imaginer un récit sacerdotal qui ne s'intéresse nullement à l'établissement concret du culte? Et est-il logique de considérer une bonne partie des versets qui accentuent le renvoi à $\mathrm{Gn}$ I, et qui soulignent le parallèle avec ce récit comme des ajouts ultérieurs? ${ }^{25}$ Cette dernière question

23 Überlieferungsgeschichte, p. 8.

${ }^{24} \mathrm{La}$ reconstruction de Pola est reprise par M. Bauks avec quelques variantes, "La signification de l'espace et du temps dans 'l'historiographie sacerdotale,", dans T. Römer (éd.), The Future of the Deuteronomistic History, BETL I47 (Leuven, 200o), pp. 2945 .

${ }_{25}$ Cf. notamment Gn I,I // Ex 39,43; Gn 2,1 // Ex 39,32; Gn 2,2 // Ex 40,33; Gn $2,3 / /$ Ex 39,43, et le tableau chez Blenkinsopp, Pentateuch, p. 218. 
se pose également quant à la démarche d'Eckart Otto. Les nombreuses divergences entre les instructions données pour la construction du sanctuaire en Ex 25-3I et le récit qui rapporte l'exécution de ces instructions (Ex 35-40) le conduisent à trouver la fin de $\mathrm{P}^{\mathrm{g}}$ en Ex 29,42b$46{ }^{26}$ Pour Otto, $\mathrm{P}$ s'efforcerait ainsi avant tout de légitimer le sacerdoce aaronide. Du coup, Otto élimine tous les versets qui opèrent une inclusion avec Gn I, ce qui paraît peu plausible. En outre, si la légitimation de la médiation sacerdotale est une préoccupation majeure de $\mathrm{P}$, il semble alors plutôt indiqué de suivre la proposition d'Erich Zenger et de chercher la fin de $\mathrm{Pg}^{\mathrm{e}}$ en $\mathrm{Lv} 8-9^{*}$, qui relate (avec cependant quelques différences par rapport à son modèle en Ex 29) la consécration d'Aaron et de ses fils. La joie et la proskynèse du peuple en $\mathrm{Lv}$ 9,24 constitueraient ainsi la fin du document sacerdotal et pourraient être interprétés comme un renvoi à $\mathrm{Gn}$ 17, I. ${ }^{27}$ Une telle hypothèse permet d'ailleurs d'intégrer dans $\mathrm{P}^{\mathrm{g}}$ certaines prescriptions sacrificielles en $\mathrm{Lv}$ I $-7^{*}$, voire même la loi de sainteté (cf. la suite $\mathrm{Ex} 40,34 \rightarrow \mathrm{Lv}$ $\mathrm{I} 7, \mathrm{I}) \cdot{ }^{28}$

\subsubsection{P" entre histoire et "loi"}

La conception de $\mathrm{P}^{\mathrm{g}}$ comme un Geschichtswerk qui ne contiendrait que du matériel narratif repose davantage sur des motivations idéologiques que sur des fondements exégétiques. Cette conception avait pour but, en réalité, de rendre à tout prix $\mathrm{P}^{\mathrm{g}}$ comparable aux autres sources du Pentateuque et de le délester de tout légalisme ou ritualisme afin de le rendre acceptable pour une certaine théologie dialectique. ${ }^{29}$ Néanmoins, la distinction rigide entre loi et récit imposée par Noth demeure, aujourd'hui encore, l'un des principaux critères pour la reconstruction de Pg. Il faudrait d'urgence reprendre cette question et, de manière générale, prêter plus d'attention à tous les textes qui sont simplement

26 "Priesterschrift," pp. $25^{-27}$ et $3^{6 .}$.

27 E. Zenger, "Die Bücher der Tora/des Pentateuch," dans Einleitung in das Alte Testament, Studienbücher Theologie I,I (Stuttgart-Berlin-Köln, 1995), pp. 34-123, ici p. 95; Id., "Priesterschrift," TRE 27 (I997), pp. 435-446, ici pp. 438-439. Cf. également le thème de la bénédiction au v. 23, qui évoque sans doute, via Ex 4o, Gn I. Et même le huitième jour de Lv 9, I pourrait faire écho à Gn 2,3.

${ }^{28}$ Cf. R.G. Kratz, Die Komposition der erzählenden Bücher des Alten Testaments. Grundwissen der Bibelkritik, UTB 2157 (Göttingen, 200o), p. Io8. Pour lui, Pg se termine en Ex 4o,34. A la suite de Noth et d'autres, Kratz attribue l'intégration de la plupart des textes du Lv à Ps; ces textes constituent ainsi des "Nachträge im Rahmen der noch selbständigen Priesterschrift" (p. II6).

${ }^{29}$ Dans ce sens Otto, "Priesterschrift," pp. 2-3. 
étiquetés "Ps". Pour cette question, le travail d'Israel Knohl peut ouvrir des perspectives intéressantes, bien que peu le suivront dans les datations préexiliques qu'il propose pour le matériel sacerdotal..$^{30} \mathrm{Knohl}$ distingue un document $\mathrm{P}$, servant à la légitimation du clergé, d'une rédaction $\mathrm{H}$ (Holinessschool), davantage préoccupée par l'éthique et la religiosité populaire. Cette rédaction $\mathrm{H}$ intègre $\mathrm{Lv}$ i7-26, mais retravaille également les textes de P, qu'elle augmente. Selon Knohl, la combinaison de $\mathrm{P}$ avec les documents non-sacerdotaux du Tétrateuque serait ainsi l'œuvre de H. L'examen de la fonction et du caractère propres des textes dits $\mathrm{P}^{\mathrm{s}} \mathrm{me}$ paraît constituer un enjeu important pour les années à venir. ${ }^{31}$

\subsubsection{Date(s) et intention(s) des textes sacerdotaux}

Il faudrait également éclaircir les questions concernant la date et l'intention de la version primitive de $\mathrm{P}$. Les débats sur l'intention de $\mathrm{P}^{\mathrm{g}}$ se focalisent souvent sur le problème de la position de la Grundschrift visà-vis du pays. A l'exception de ceux qui retrouvent la fin de $\mathrm{P}$ dans quelques fragments en Jos, la majorité des chercheurs s'accordent pour dire que $\mathrm{P}$ n'aurait pas raconté l'installation d'Israël dans son pays. Or Ex 6,2-8, qui est unanimement reconnu comme un texte clé de $\mathrm{P}$, se termine par la promesse suivante: "Je vous ferai entrer dans le pays que, la main levée, j'ai donné à Abraham, à Isaac et à Jacob. Je vous le donnerai en possession". On peut bien sûr décréter que ce verset appartient à une rédaction ultérieure, ${ }^{32}$ puisqu'il semble présupposer et corriger $\mathrm{Ez} 20$ et $\mathrm{Ez} 33,24$, mais aucun critère traditionnel de la Literarkritik ne nécessite une telle opération (d'autant plus que Ex 6,8 fait allusion à $\mathrm{Gn}$ 17,8). Une solution possible serait de trouver l'"aboutissement" de Gn 17 et d'Ex 6 en Ex 29,45-46, à condition de voir dans ces versets l'annonce de l'établissement du culte dans le temple de Jérusalem. Dans la perspective de $\mathrm{P}$, c'est essentiellement le culte, et non la possession du pays, qui garantit la présence divine au sein de la communauté. Certes,

${ }^{30}$ I. Knohl, The Sanctuary of Silence. The Priestly Torah and the Holiness School (Minneapolis, 1995).

31 On constate ces dernières années un intérêt grandissant pour $\mathrm{P}$, mais qui se limite toutefois presque exclusivement à la fameuse Grundschrift.

${ }^{32}$ F. Kohata, Fahwist und Priesterschrift in Exodus 3-14, BZAW I66 (Berlin, New York, I986), pp. 31-34; B. Gosse, "Exode 6,8 comme réponse à Ezéchiel 33,24," RHPhR 74 (I994), pp. 24I-247; p. 246: "en conformité avec la rédaction d'ensemble du Pentateuque". 
la participation effective du peuple au culte requiert l'entrée dans le pays, et, en ce sens, la communauté idéale pour $\mathrm{P}$ est celle rassemblée autour du temple de Jérusalem. Mais contrairement au courant dtr, le pays ne constitue pas pour $\mathrm{P}$ un enjeu géopolitique, il n'est que le cadre à l'intérieur duquel la présence cultuelle de Yhwh peut se réaliser. ${ }^{33} \mathrm{La}$ recherche actuelle converge d'ailleurs dans l'idée que la théophanie au Sinaï constitue le "centre" de l'œuvre sacerdotale.

Reste encore le problème de la datation de $\mathrm{Pg}$. Contrairement à plusieurs auteurs, je ne crois pas que la localisation du sanctuaire au Sinaï soit un argument convaincant pour dater $\mathrm{P}^{\mathrm{g}}$ avant la reconstruction du temple. La dépendance de $\mathbf{P}$ vis-à-vis du livre d'Ezéchiel suggère au contraire une date plus tardive. ${ }^{34}$ L'effort de $\mathrm{P}$ consiste de toute façon à enraciner toutes les institutions décisives dans les "origines". Le Sabbat est fondé lors de la création du monde, les lois alimentaires à l'époque de la nouvelle humanité, la circoncision sous Abraham, et le culte d'Israël aussitôt que Yhwh s'est révélé sous son vrai nom. En spéculant, on pourrait même se demander si la transposition du sanctuaire au Sinaï ne signifie pas également de la part de $\mathrm{P}$ une certaine acceptation d'un sanctuaire yahwiste ailleurs qu'à Jérusalem, par exemple à Bethel ou à Samarie. ${ }^{35}$

Dans tous les cas, une dernière chose mérite d'être soulignée: si la version $\mathrm{P}$ s'est terminée quelque part dans la péricope du Sinai, il faut renoncer à l'idée que c'est cette version qui aurait constitué l'ossature du Pentateuque, et qui aurait servi à en définir l'étendue.

${ }^{33}$ Cf. M. Köckert, "Das Land in der priesterlichen Komposition des Pentateuch," dans D. Vieweger et E.-J. Waschke (éd.), Von Gott reden. Beiträge zur Theologie und Exegese des Alten Testaments. Festschrift für Siegfried Wagner zum 65. Geburtstag (Neukirchen-Vluyn, I995), pp. I47-162, spéc. 152-153.

${ }_{34}$ On ne peut guère attribuer $\mathrm{Ez} 2 \mathrm{O}$ au prophète Ezechiel, comme le fait Pola; sur ce chapitre, cf. T. Krüger, Geschichtskonzepte im Ezechielbuch, BZAW ı8o (Berlin, New York, I989); F. Sedlmeier, Studien zur Komposition und Theologie von Ezechiel 2o, SBB 2I (Stuttgart, 1990).

${ }^{35}$ B. Diebner, "Gottes Welt, Moses Zelt und das salomonische Heiligtum. Anmerkungen zur Vollendung des Tempels," dans T. Römer (éd.), Lectio difficilior probabilior? L'exégèse comme expérience de décloisonnement. Mélanges offerts à Françoise Smyth-Florentin B.DBAT I2 (Heidelberg, I99I), pp. I27-I54; J. Blenkinsopp, "The Judaean Priesthood during the Neo-babylonian and Achaemenid Period," CBQ 6o (1998), pp. 25-43. P serait donc moins préoccupé par la centralisation que D. 


\section{La renaissance de l'Hexateuque}

\section{I. De la contestation de l'histoire dtr à la redécouverte de l'Hexateuque}

Comme Otto l'a souligné avec raison, la nouvelle critique du Pentateuque s'est fort peu occupée du livre du Deutéronome. ${ }^{36}$ Ceci s'explique principalement par le fait que dans la suite des travaux de Noth, le dernier livre du Pentateuque était surtout envisagé en tant qu'ouverture de l'histoire dtr, ce qui tendait de fait à transformer le Pentateuque en Tétrateuque. ${ }^{37}$ Depuis quelques années, l'existence d'une histoire dtr (peu importent ici ses différentes variantes) est contestée avec virulence. ${ }^{38} \mathrm{~A}$ mon avis, ces contestations ne nécessitent pas (encore) l'abandon de la thèse de Noth. ${ }^{39}$ Elles ont pourtant le mérite de reposer un certain nombre de questions qui, suite au triomphe de la théorie de l'histoire dtr, avaient été quelque peu occultées, et ceci vaut notamment pour la théorie de l'Hexateuque.

Ce n'est certainement pas un hasard si la mise en question de l'existence d'une histoire dtr s'accompagne du retour d'un Hexateuque. Car il est évident qu'un certain nombre de textes dans le Pentateuque demandent une suite dans le livre de Josué. Un exemple classique est le thème de la promenade des ossements de Joseph en Gn 50,25 et en Ex 13,19, qui ne trouve son accomplissement qu'en Jos 24. On notera d'ailleurs à ce propos que la fin de Jos 24 est clairement construite en parallèle à la finale du livre de la Genèse. Selon Jos 24,29 Josué meurt âgé de IIo ans, tout comme Joseph en Gn 50,26. Mais il existe encore

${ }^{36}$ E. Otto, Das Deuteronomium im Pentateuch und Hexateuch. Studien zur Literaturgeschichte von Pentateuch und Hexateuch im Lichte des Deuteronomiumsrahmen, FAT 30 (Tübingen, 2000), pp. I-3.

37 Cf. récemment J. Van Seters, The Pentateuch: A Social Science Commentary, Trajectories (Sheffield, 1999), pp. 93-99.

${ }^{38}$ Cf notamment C. Westermann, Die Geschichtsbücher des Alten Testaments. Gab es ein deuteronomistisches Geschichtswerk? ThB.AT 87 (Gütersloh, I994); E.A. Knauf, "L'historiographie deutéronomiste' (DtrG) existe-t-elle?," dans A. de Pury, T. Römer et J.-D. Macchi (éd.), Israël construit son histoire. L'historiographie deutéronomiste à la lumière des recherches récentes, Le Monde de la Bible 34 (Genève, 1996), pp. 409-418; H.N. Rösel, Von Josua bis Jojachin. Untersuchungen zu den deuteronomistischen Geschichtsbüchern des Alten Testaments, VT.S 75 (Leiden, Boston, Köln, I999); R. G. Kratz, "Der literarische Ort des Deuteronomiums," dans R.G. Kratz et H. Spieckermann (éd.), Liebe und Gebot. Studien zum Deuteronomium. Festschrift zum 7o. Geburtstag von Lothar Perlitt, FRLANT I9o (Göttingen, 2000), pp. IOI-1 20.

${ }^{39}$ Cf. à ce sujet T. Römer, "La fin de l'historiographie deutéronomiste et le retour de l'Hexateuque?," ThZ 57 (200I), pp. 269-280. 
bien d'autres textes dans le Pentateuque qui présupposent l'existence d'un Hexateuque; ainsi par exemple le rôle de Caleb en $\mathrm{Nb}^{\mathrm{I}} 3^{-\mathrm{I}} 4$ (cf. Jos I4,I3-I5) ou le début de l'époque de la manne en Ex I6,35, époque qui se termine en Jos 5, I2. Dans le cadre de l'hypothèse dtr, on expliquait de tels textes soit par un élargissement de l'histoire dtr, soit par une rédaction ou une "composition" dtr du Tétrateuque (Rudolf Smend, Jacques Vermeylen, Erhard Blum) ${ }_{,}^{40}$ soit par un prologue yahwiste qui n'aurait pas seulement fait précéder l'histoire dtr par l'adjonction de $\mathrm{Gn}-\mathrm{Nb}^{*}$ mais qui serait également intervenu dans le livre de Josué (Van Seters). ${ }^{41}$ Mais ces modèles peinent cependant à rendre compte du fait que Jos 24 veut visiblement instituer une coupure importante par rapport aux livres suivants. ${ }^{42}$

\subsection{Un Hexateuque à la Wellhausen?}

Une alternative à ce problème consiste alors, pour certains auteurs, à retourner au maître Wellhausen et à postuler un Hexateuque ancien. Ainsi Reinhard Kratz avance l'hypothèse d'un "Hexateuque" pré-deutéronomique, qui n'aurait cependant commencé qu'en Ex 2,I et se serait terminé en Jos I2,1a. 9-24. Une telle hypothèse soulève toutefois un certain nombre de problèmes. Pour aboutir à un fil narratif cohérent, Kratz reconstruit pour la fin de son Hexateuque la succession suivante: Nb 25,Ia; Dt 34,5-6; Jos 2, Iss.; 3,1.14a. I6; 4,Igb; 6*; 8*; 12,Ia. $9-24 .^{43}$ Il faut d'abord accepter le fait que certains versets qui, à

40) R. Smend, Die Entstehung des Alten Testaments (Stuttgart et al., 1978), pp. 62-69; J. Vermeylen, "L'école deutéronomiste aurait-elle imaginé un premier canon des Ecritures?," dans T. Römer (éd.), The Future of the Deuteronomistic History, BETL I47 (Leuven, 2000), pp. 223-240; Blum, Studien, passim.

41 J. Van Seters, "Joshua 24 and the Problem of Tradition in the Old Testament," dans W.B. Barrick et J.R. Spencer (éd.), In the Shelter of Elyon. Essays on Ancient Palistinian Life and Literature in Honor of G.W. Ahlström, JSOT.S 31 (Trowbridge, I984), pp. I39158; "The Deuteronomistic Redaction of the Pentateuch: The Case Against It," dans M. Vervenne et J. Lust (éd.), Deuteronomy and Deuteronomic Literature. Festschrift C.H.W. Brekelmans, BETL i33 (Leuven, I997), pp. 301-319.

${ }^{42}$ Blum a donc tout à fait raison de parler d'une "Jos 24-Redaktion," mais les motivations de celle-ci demeurent quelque peu obscures; cf. en dernier lieu E. Blum, "IDer kompositionelle Knoten am Übergang von Josua zu Richter. Ein Entflechtungsvorschlag," dans M. Vervenne et J. Lust (éd.), Deuteronomy and Deuteronomic Literature. Festschrift C.H.W. Brekelmans, BETL 133 (Leuven, 1997), pp. 181-212.

${ }^{43}$ Kratz, Komposition der erzählenden Bücher, pp. 221 et 303. Pour un "ancien" Hexateuque cf. également C. Minette de Tillesse, "La Crise du Pentateuque," ZAW III (1999), pp. 1-9. 
l'origine, étaient destinés à se suivre ( $\mathrm{Nb} 25$, Ia et $\left.\mathrm{Dt} 34,5^{-6}\right)$ se trouvent maintenant séparés l'un de l'autre par une quarantaine de chapitres. En outre, pour obtenir un "Hexateuque " (que Kratz appelle "E") de l'époque monarchique (au VIIème siècle), Kratz est obligé de considérer des textes comme Dt $34,5^{-6^{44}}$ ou Jos 2 comme étant pré-dtr, ce qui paraît difficilement défendable tant sur le plan stylistique que rédactionnel..$^{45}$ La tentative de renouer avec l'idée d'un Hexateuque à la Wellhausen semble donc plutôt hasardeuse. Il faut pourtant essayer de rendre compte des signaux présents dans le Pentateuque et dans le livre de Josué qui indiquent la volonté de créer un Hexateuque.

\subsection{Un Hexateuque à l'époque perse?}

Un des textes décisifs pour cette question est le second discours d'adieu de Josué en Jos 24, puisque c'est ce résumé de l'histoire placé dans la bouche de Josué qui crée en quelque sorte un Hexateuque, en racontant l'histoire des pères au-delà du fleuve jusqu'à la conquête du pays. Bien que les opinions au sujet de ce chapitre soient encore multiples, l'idée d'une datation postexilique et post-dtr pour Jos 24 semble faire son chemin. ${ }^{46}$ Cette conclusion implique, si on l'adopte, que les traces d'un Hexateuque dans la Bible hébraïque ne sont pas un donné ancien, mais témoignent d'un projet tardif, concurrent à celui du Pentateuque. On peut ainsi envisager une coalition de certains milieux dtr et sacerdotaux qui, au moment de la publication de la Torah, désiraient y inclure le livre de Josué. En particulier, l'hypothèse d'une telle coalition expliquerait aisément la présence de certains textes mixtes en Jos (par ex. Jos 3-4 ou 6) où une séparation entre "P" et " $D$ " par les moyens de la critique littéraire ne semble guère possible. Selon Jos 24,26, Josué écrit un livre comme Moïse en Dt 31,24 ("et Josué écrivit toutes ces paroles dans le livre de la Torah de Dieu”). L'expression ספר תורת אלהים ne se

${ }^{44}$ Ainsi dans Komposition der erzählenden Bücher. Dans "Ort," p. Io2 et p. I19, n. 73, Kratz précise que le v. 5 primitif n'aurait contenu que les mots "et Moïse mourut làbas". L'argument est néanmoins complètement circulaire. Kratz reconnaît que le verset est marqué par la terminologie dtr; mais il tient cette terminologie pour secondaire parce qu'il affirme qu'une version pré-dtr de Dt $34^{*}$ a dû exister.

${ }^{45}$ Pour Dt 34,5-6, cf. les remarques critiques d'Otto, Deuteronomium, pp. 224-225, n. 29I. Jos 2 est un texte post-dtr qui interrompt la chronologie de Jos I et 3, cf. J. Van Seters, In Search of History: History in the Ancient World and the Origin of Biblical History (New Haven-London, 1983), p. 325.

${ }^{46} \mathrm{Cf}$. notamment M. Anbar, Josué et l'alliance de Sichem (fosué 24:I-28), BET 25 (Frankfurt/M. et al., I992). 
retrouve dans toute la Bible hébraïque qu'en $\mathrm{Ne}$ 8, I8: "On lut dans le livre de la Loi de Dieu (ספר תורת האלהים)"; et Ne 8,I7 souligne explicitement que cette lecture publique s'inscrit dans le cadre de la première célébration de la fête des huttes depuis l'époque de Josué. Cette observation suggère la volonté d'intégrer Josué dans cette Torah. ${ }^{47}$ On pourrait alors supposer que ספר תורת אלהים était le nom donné à l'Hexateuque, tandis que ספר) תורת משה (ספר), qui signifiait d'abord le Dt (IR 2,3; 2 R I4,6; 23,25), servit par la suite à désigner le Pentateuque. ${ }^{48} \mathrm{Il}$ est donc tout à fait possible que le Pentateuque et l'Hexateuque aient coexisté durant un certain temps comme le pense également Otto. ${ }^{49}$ Le conflit entre Penta- et Hexateuque est d'ailleurs également reflété en Dt 34. Nous avons vu que les v. 7-9 ne peuvent être attribué à Pg; ils relèvent plutôt d'une rédaction de type "Hexateuque", puisqu'ils insistent sur le lien étroit entre Moïse et Josué, et qu'ils font (à l'instar de Jos 24) de Josué un deuxième Moïse. Les v. Io-I2 appartiennent par contre à la rédaction du Pentateuque, qui corrige cette vue en soulignant le caractère absolument incomparable de Moïse. En ce sens, le texte de Dt 34,IO-I2 dit clairement que la Torah d'Israël se définit exclusivement par le biais de la médiation mosaïque.

Otto est certainement l'avocat le plus fervent de la coexistence, dans la Torah, d'une rédaction "Hexateuque" et d'une rédaction "Pentateuque". Il situe ces deux rédactions entre la seconde moitié du cinquième siècle et le début du quatrième siècle et voit dans la rédaction Hexateuque la tentative de corriger un certain "golahcentrisme", qui se reflète par exemple dans le livre d'Ezéchiel et qui est également sous-jacent dans la rédaction "Pentateuque" (rédaction qu'il ne faut pas confondre avec la rédaction finale du Pentateuque). ${ }^{50}$ Otto a précisé sa position à partir d'une exégèse de $\mathrm{Nb}_{1} 3^{-14}$ et du récit parallèle de Dt I,Igss. Selon lui, il existerait en $\mathrm{Nb}_{1} 3^{-1}{ }^{-1}$ une Grunderzählung préexilique, qui aurait servi de base au rédacteur de Dt I (rédacteur qu'il identifie, à la suite de Norbert Lohfink,,$^{51}$ à "DtrL"). Dans cette hypo-

${ }^{47} \mathrm{La}$ grande joie de $\mathrm{Ne} 8, \mathrm{I} 7$ renvoie à $\mathrm{Dt}$ r6, I4 (dtr) et la commémoration du huitième jour en 8,18 renvoie à $\operatorname{Lv} 23,36$.

${ }^{48}$ Cf. pour ceci et pour la suite T.C. Römer et M.Z. Brettler, "Deuteronomy 34 and the Case for a Persian Hexateuch," $7 B L$ i I9 (200o), pp. 40I-4I9.

${ }^{49}$ Deuteronomium, p. 231.

50 Deuteronomium, pp. 26r-263.

51 N. Lohfink, "Kerygmata des Deuteronomistischen Geschichtswerks," dans J. Jeremias et L. Perlitt (éd.), Die Botschaft und die Boten. FS H.W. Wolff (Neukirchen-Vluyn, I98I), pp. 87-100 = Studien zum Deuteronomium und zur deuteronomistischen Literatur. II, SBAB 12 (Stuttgart, I99I), pp. I25-142. 
thèse, les textes de $\mathrm{Nb}$ I3-I4 qui ont souvent été attribués à $\mathrm{P}$ ou à des ajouts post-P proviendraient en réalité des rédactions "Hexateuque" et "Pentateuque". ${ }^{52}$ Cette solution signifie toutefois que plus des trois quarts du récit de $\mathrm{Nb}_{13}{ }^{-}{ }^{-14}$ sont attribués à ces deux rédactions, alors que le statut du récit de base reste relativement flou. ${ }^{53}$ Ceci étant dit, la démarche d'Otto est fort stimulante, et elle ouvre de nouvelles pistes pour la recherche sur le Pentateuque.

\subsection{Le Deutéronome entre Hexateuque et Tétrateuque}

Dans la situation actuelle, le Deutéronome demeure le livre du Pentateuque dont les origines font l'objet d'un certain consensus, et peuvent être situées à l'époque assyrienne. ${ }^{54}$ Néanmoins, l'un des principaux problèmes concerne l'existence des récits parallèles en Dt I-3; 9-1o et en Ex-Nb. Dans l'hypothèse d'une histoire dtr, Dt I-3 avait pour fonction de récapituler certains événements centraux du Tétrateuque. La nouvelle critique, qui présupposait également l'existence d'une histoire dtr (cf. notamment Van Seters, Rose), a inversé la relation de dépendance entre Tétrateuque et Deutéronome, puisqu'elle considère, souvent avec des arguments convaincants, les récits de $\mathrm{Nb}$ comme des élaborations ultérieures des traditions conservées dans le Dt. Pour ceux qui ont cessé de croire à la thèse de Noth, la question de la fonction et de la signification de ces chapitres dans le Deutéronome se pose à nouveau. Selon Kratz, ${ }^{55} \mathrm{Dt} \mathrm{I}-3^{56}$ aurait été créé pour insérer le livre du Dt*, qui aurait d'abord connu une histoire rédactionnelle indépendante, entre $\mathrm{Nb}$ 25, I et Dt 34, I.5.6 dans un "Hexateuque" déjà existant. Une telle explication paraît peu convaincante, puisque du simple point de vue de la cohérence narrative, il paraît inutile et superflu de répéter les récits

${ }^{52}$ Otto, Deuteronomium, pp. 26ss.

${ }^{53}$ Fait-il partie de la "Mose-Grunderzählung" du VIIème siècle? Cf. E. Otto, "Mose und das Gesetz. Die Mose-Figur als Gegenentwurf Politischer Theologie zur neuassyrischen Königsideologie im 7. Jh. v. Chr.," dans E. Otto (éd.), Mose. Ägypten und das Alte Testament, SBS 189 (Stuttgart, 200o), pp. $42-83$.

${ }^{54} \mathrm{La}$ recherche récente a souligné de nombreux parallèles entre le Dt (primitif) et les textes de propagande et de loi assyriens; cf. entre autres H.U. Steymans, Deuteronomium 28 und die adê zur Thronfolgeregelung Asarhaddons. Segen und Fluch im Alten Orient und in Israel, OBO 145 (Fribourg CH, Göttingen, I995); E. Otto, Das Deuteronomium. Politische Theologie und Rechtsreform in fuda und Assyrien, BZAW 284 (Berlin, New York, 1999).

${ }^{55}$ Kratz, "Ort des Deuteronomiums," p. Iog.

36 Nous laissons de côté la question de la diachronie de ces chapitres qui est remarquablement complexe. 
immédiatement précédents. L'existence des parallèles en $\mathrm{Ex}, \mathrm{Nb}$ et en Dt reste un argument fort pour l'existence d'une histoire dtr commençant en Dt I-3. Mais il est également clair que l'origine et la fonction des récits de révolte en $\mathrm{Nb}$ demande de nouvelles investigations. ${ }^{57}$

\section{Yahwiste, composition D ou théorie des fragments?}

\section{I. Yahwiste et "composition D": le problème de leurs sources et de leur profil}

La distinction entre du matériel $\mathrm{P}$ et du matériel non-P reste toujours un point de départ valable. Les différences d'appréciation apparaissent pourtant très vite lorsqu'il s'agit d'identifier plus précisément la nature de ce matériel. Van Seters attribue la plupart des textes non sacerdotaux à son Yahwiste post-dtr, qu'il considère comme un Hérodote hébraïque. Ce Yahwiste est essentiellement un auteur, au sens fort du terme, dont les sources, s'il en a utilisées, ne peuvent de toute manière plus être reconstruites. ${ }^{58}$ Christoph Levin défend également la thèse d'un Yahwiste exilique, ${ }^{59}$ qu'il situe entre le Dt et l'historiographie dtr. Contrairement à Van Seters, il se livre à une critique littéraire qui rappelle celle du XIXème siècle. Pour Levin, le Yahwiste n'est pas un auteur mais un rédacteur, qui aurait eu à sa disposition de nombreuses sources; il serait ainsi le créateur du Tétrateuque (dont la fin se trouverait en Nb 22-24). A l'opposé de Van Seters, Levin est confiant dans la possibilité de reconstruire ces sources $\left(\mathrm{J}^{2}\right)$, et il envisage également un nombre important de suppléments post-yahwistes $\left(J^{\mathrm{S}}\right)$. Pour lui, $\mathrm{J}$ est un théologien de la diaspora, qui valorise une religiosité populaire et qui polémique contre l'idéologie deutéronomique du sanctuaire unique. Van Seters et Levin s'accordent dans leur conception de "J" comme étant un théologien plutôt "libéral", ${ }^{60}$ qui se trouverait en opposition

57 A priori, les arguments pour une dépendance des textes du Tétrateuque par rapport à Dt I-3 me paraissent toujours assez convaincants, cf. notamment Van Seters, "Deuteronomistic Redaction"; Aurelius, Fürbitter, p. I34; J. Blenkinsopp, "Deuteronomic Contribution to the Narrative in Genesis-Numbers: A Test Case," dans L.S. Schearing et S.L. McKenzie (éd.), Those Elusive Deuteronomists: The Phenomenon of Pan-Deuteronomism, JSOT.S 268 (Sheffield, I999), pp. 84-1I5.

${ }^{58}$ Cf. notamment J. Van Seters, Prologue to History: The Yahwist as Historian in Genesis (Zürich, I992); id., The Life of Moses: The Yahwist as Historian in Exodus-Numbers (Louisville, Kampen, I994).

59 C. Levin, Der fahwist, FRLANT 157 (Göttingen, 1993).

${ }^{60}$ J. Van Seters, "The Theology of the Yahwist. A Preliminary Sketch," dans I. Kottsieper et al. (éd.), "Wer ist wie du, HERR, unter den Göttern.?". Studien zur Theologie und Reli- 
à l'orthodoxie deutéronomique ou deutéronomiste; alors que Rose et d'autres auteurs voient dans le Yahwiste un descendant direct des Deutéronomistes. ${ }^{61}$

Ces positions opposées font apparaître un certain nombre de problèmes de méthode qui concernent d'abord la définition du terme "dtr", mais aussi la question de la cohérence de l'œuvre yahwiste. Les mêmes questions se posent évidemment pour les tenants de l'hypothèse d'une composition $\mathrm{D}$, laquelle dépend bien sûr également, d'une manière ou d'une autre, de l'histoire dtr (Blum, Rainer Albertz, et dans un certain sens également David Carr $\left.{ }^{62}\right)$. En ce qui concerne la caractérisation et l'identification d'un texte dtr, on ne peut pas se contenter de simples statistiques de style et de vocabulaire. Pour des mots peu fréquents, les résultats sont aléatoires; en outre, le style dtr est facilement imitable, et on le retrouve jusque dans les écrits néotestamentaires. ${ }^{63} \mathrm{Il}$ faut donc nécessairement compléter les enquêtes statistiques par une analyse des techniques rédactionnelles, ${ }^{64}$ et surtout par une analyse de l'idéologie sous-jacente à ces textes; bien que cette dernière démarche soit délicate, elle n'en est pas moins indispensable. Si pour décrire la visée théologique de $\mathrm{J}$ (ou même de la composition D) l'accent principal est mis sur la Genèse, et plus précisément sur les récits patriarcaux, il n'est guère étonnant que le Yahwiste apparaisse plutôt en opposition à la pensée dtr. Si par contre on met en avant certaines péricopes en Exode ou en Nombres, comme par exemple Ex 23,31-33 ou 34,10-13 (une version "militaire" de la promesse du pays, liée à l'expulsion des peuples autochtones) ${ }_{, 65}^{65}$ on peut en effet postuler une certaine proximité

gionsgeschichte Israels für Otto Kaiser zum 7o. Geburtstag (Göttingen, 1994), pp. 219-230.

${ }_{61}$ Cf. M. Rose, Deuteronomist und Jahwist. Untersuchungen zu den Berührungspunkten beider Literaturwerke, AThANT 67 (Zürich, I98I); cf. p. 326 ("weiterführt und vertieft"); H.C. Schmitt, "Die Erzählung vom Goldenen Kalb Ex. $32^{*}$ und das Deuteronomistische Geschichtswerk," dans S. L. McKenzie et T. Römer (éd.), Rethinking the Foundations. Historiography in the Ancient World and in the Bible: Essays in Honour of John Van Seters, BZAW 294 (Berlin, New York, 200o), pp. 235-250; ici pp. $237^{-2}-20$.

62 D.M. Carr, Reading the Fractures of Genesis (Louisville KY, 1996). Carr se contente cependant de parler d'une rédaction semi-dtr, qui aurait édité une sorte de "ProtoPentateuque" au VIème siècle.

${ }^{63}$ Cf. l'étude magistrale de O.H. Steck, Israel und das gewaltsame Geschick der Propheten. Untersuchungen zur Überlieferung des deuteronomistischen Geschichtsbildes im Alten Testament, Spätjudentum und Urchristentum, WMANT 23 (Neukirchen-Vluyn, I967).

${ }^{64}$ Cf. Ska, "Le Pentateuque: état de la recherche," pp. $256-257$.

${ }^{65}$ L.evin, Jahwist, pp. 366-369, considère l'ensemble de 20,24-23,33 et de 34,9b27 comme post-J; de même, Blum, Studien, pp. 365-369, attribue ces textes à des 
avec l'idéologie dtr. Cette différence d'appréciation ne réside-t-elle pas dans le fait que la Genèse est moins marquée par le style et par l'idéologie dtr que les livres de l'Exode et des Nombres? Cette question nous introduit à une problématique plus large, qui concerne la redécouverte, dans la recherche récente, de l'indépendance relative de certaines "unités majeures".

\subsection{Le retour d'une théorie des fragments?}

\subsection{Le lien entre l'histoire des Patriarches et l'Exode}

En terminant notre histoire de la recherche sur le Pentateuque en I989, Albert de Pury et moi-même avions envisagé la possibilité que Gn I235 et Ex-Dt* (Jos?) représentent deux versions concurrentes des origines du peuple d'Israël. ${ }^{66}$ En effet, les travaux menés par de Pury ont établi que le cycle de Jacob en Gn $25^{-} 36^{*}$ constitue un mythe autonome des origines d'Israël, qui semble en outre se trouver en concurrence avec la tradition de la sortie d'Egypte. Ce conflit voit s'affronter une conception généalogique ("populaire") des origines et une conception vocationnelle (de type "prophético-deutéronomiste"). Il dut être virulent à l'époque de l'exil, comme le suggère Ez 33,24. L'une des raisons pour lesquelles cette thèse d'un affrontement des mythes d'origine reste peu discutée est liée au fait, déjà souligné, que le livre du Deutéronome est insuffisamment pris en considération dans le débat actuel sur le Pentateuque. En effet, si l'hypothèse selon laquelle les références fréquentes aux "pères" dans le Dt ainsi que dans l'historiographie dtr ne renvoient pas aux traditions patriarcales mais aux origines égyptiennes d'Israël (cf. Van Seters et Römer ${ }^{67}$ ) s'avère justifiée, cela signifierait que la rédaction dite "dtr" dans le Pentateuque ne comprenait probablement pas les récits de la Genèse. Du coup, la question se pose de savoir à qui il faut attribuer le lien entre ces deux ensembles littéraires.

Konrad Schmid a repris cette question, et est arrivé à la conclusion que $\mathrm{P}$ serait le premier à avoir effectué un lien littéraire entre la

\footnotetext{
interventions rédactionnelles sur la composition D. Van Seters, Life of Moses, p. 355, attribue les deux textes au "Yahwiste," en soulignant comme Blum et Levin leur dépendance littéraire par rapport à des textes tels que Dt $7, \mathrm{I}-5$.

66 A. de Pury, T. Römer, "Pentateuque," p. 77

${ }^{67}$ J. Van Seters, "Confessional Reformulation in the Exilic Period," VT 22 (1972), pp. 448-459; T. Römer, Israels Väter. Untersuchungen zur Väterthematik im Deuteronomium und in der deuteronomistischen Tradition, OBO 99 (Freiburg CH, Göttingen, 199o), passim.
} 
Genèse et les traditions sur Moïse. ${ }^{68}$ Il considère que $\mathrm{Ex}{ }_{2-\mathrm{I}} \mathrm{R}$ I2* aurait existé d'abord comme une légende d'origine et de légitimation du Royaume du Nord qui aurait été transformée après la destruction de Jérusalem en une "grande doxologie de jugement expliquant la chute de Juda et de Jérusalem". ${ }^{69}$ Puisque Schmid considère P comme une selbstständige Quellenschrift, ${ }^{70}$ il s'ensuit que ce sont des rédacteurs postérieurs (auxquels il faut attribuer des textes comme Gn I5; Ex 3-4; Jos 24) qui font le lien entre le matériel $\mathrm{P}$ et les traditions non sacerdotales. Ici, les thèses de Schmid rejoignent celles d'Otto pour qui ce sont les rédactions du Pentateuque, voire de l'Hexateuque, qui ont façonné la Torah.

La thèse de Schmid est séduisante, bien qu'elle mérite discussion et approfondissement. Certains textes de facture dtr (et d'origine présacerdotale?) semblent présupposer au minimum l'existence d'un lien entre une tradition de descente en Egypte et la sortie d'Egypte, comme Dt $26,5^{-9}$ ou i $\mathrm{S}$ i2,8ss. On peut bien sûr envisager pour ces textes une datation post-sacerdotale, ${ }^{71}$ mais c'est aussi courir le risque d'un raisonnement circulaire. Il est également évident que certains textes dans les récits patriarcaux contiennent des allusions souvent ironiques à la tradition de l'Exode, notamment en Gn 12,10-20 ou en Gn $16 .{ }^{72}$ Cela signifie que les récits de l'Exode étaient déjà connus pour une partie des auteurs des récits patriarcaux, qui voulaient apparemment s'en distancier, voire les critiquer.

La question du lien entre la Genèse et l'Exode ne peut être débattue sans une prise de position concernant le roman de Joseph. Sur l'ori-

${ }^{68}$ K. Schmid, Erzväter und Exodus. Untersuchungen zur doppelten Begründung der Ursprünge Israels innerhalb der Geschichtsbücher des Alten Testaments, WMANT 8I (Neukirchen-Vluyn, 1999). Cf. également J.C. Gertz, Tradition und Redaktion in der Exoduserzählung. Untersuchungen zur Endredaktion des Pentateuch, FRLANT I86 (Göttingen, 1999), pp. 384-391; et Römer, Väter, p. 547.

${ }^{69}$ Erzväter, p. 163.

${ }^{70}$ Il explique que "'P' mit ihrer Abfolge von Erzvätern und Exodus nur als urprünglich selbständige Quelle sinnvoll vorzustellen ist" (p. 372).

${ }^{71}$ J.C. Gertz, "Die Stellung des kleinen geschichtlichen Credos in der Redaktionsgeschichte von Deuteronomium und Pentateuch," dans R.G. Kratz et H. Spieckermann (éd.), Liebe und Gebot. Studien zum Deuteronomium. Festschrift zum 7o. Geburtstag von Lothar Perlitt, FRLANT igo (Göttingen, 200o), pp. 30-45.

72 Cf. par ex. T.B. Dozemann, "The Wilderness and Salvation History in the Hagar Story," $\mathcal{F B L} 117$ (1998), pp. 23-43; W. Oswald, "Die Erzeltern als Schutzbürger. Überlegungen zum Thema von Gen 12,10-20 mit Ausblick auf Gen 20.21,22-34 und Gen 26," $B \mathcal{N} 106$ (2001), pp. 79-89. 
gine et la fonction de ce récit, il n'existe aucun consensus. Les dates proposées actuellement vont du IXème siècle (légitimation du royaume du Nord) jusqu'à l'époque hellénistique. ${ }^{73}$ Il me semble pourtant que l'absence de l'histoire de Joseph de tous les sommaires historiques de la Bible hébraïque, à l'exception du Ps 105, et même de l'éloge des Pères du Siracide ${ }^{74}$ encourage à y voir un écrit de diaspora tardif, ${ }^{75}$ d'autant plus qu'on peut parfaitement reconstruire un récit de transition entre Gn et Ex, d'origine sacerdotale, qui ne contienne pas l'histoire de Joseph. ${ }^{76}$ Dans tous les cas, l'histoire de Joseph n'est pas nécessaire pour établir un lien entre les Patriarches et l'Exode.

\subsubsection{L'histoire des origines ( $\left.G n_{I-I I}\right)$}

Dès 1981, Frank Crüsemann avait insisté sur l'indépendance de la version présacerdotale du récit des origines, dont il faisait remarquer qu'elle ne contient aucun indice qui laisse présager une suite en Gn I2-25*. ${ }^{77}$ Le seul lien clair entre les deux ensembles littéraires se trouve en Gn I2,I-3, mais il s'agit-là d'un raccord tardif, qu'une étude récente de Ska considère comme postsacerdotal. ${ }^{78}$ L'enquête récente de Markus Witte va dans la même direction. Selon lui, il n'existe pas de rédaction présacerdotale qui aurait relié Gn $2-8^{*}$ au reste du Pentateuque. Ce lien serait uniquement l'œuvre d'un rédacteur final, dont le travail

${ }^{73}$ Cf. notamment W. Dietrich, Die Josephserzählung als Novelle und Geschichtsschreibung. Zugleich ein Beitrag zur Pentateuchfrage, Biblisch theologische Studien 14 (NeukirchenVluyn, I989); B.J. Diebner, "Le roman de Joseph, ou Israël en Egypte. Un midrash post-exilique de la Tora," dans O. Abel et F. Smyth (éd.), Le livre de traverse. De l'exégèse biblique à l'anthropologie, Patrimoines (Paris, I992), pp. 55-7I; A. Catastini, Storia di Giuseppe (Genesi 37-50) (Venezia, 1994). Pour l'histoire de la recherche, cf. H. Seebass, Genesis III. Josephgeschichte (37,1-50,26) (Neukirchen-Vluyn, 2000), pp. 209-219.

${ }^{74}$ Joseph n'est pas mentionné entre Jacob et Moïse en Sir 44,23 et 45, I. Il apparaît seulement (comme ajout?) en 49,15.

${ }^{75}$ Cf. récemment C. Uehlinger, "Fratrie, filiations et paternités dans l'histoire de Joseph (Genèse 37-50*)," dans J.-D. Macchi et T. Römer (éd.), Jacob. Commentaire à plusieurs voix de Gen. 25-36. Mélanges offerts à Albert de Pury, Le Monde de la Bible 44 (Genève, 200I), pp. 303-328, qui postule une première version au VIème siècle.

76 T. Römer, "La narration, une subversion. L'histoire de Joseph (Gn 37-5o) et les romans de la diaspora," dans G.J. Brooke et J.-D. Kaestli (éd.), Narrativity in Biblical and Related Texts, BETL 149 (Leuven, 200o), pp. I7-29 spéc. 23.

${ }^{77}$ F. Crüsemann, "Die Eigenständigkeit der Urgeschichte. Ein Beitrag zur Diskussion um den 'Jahwisten'," dans J. Jeremias et L. Perlitt (éd.), Die Botschaft und die Boten. FS H.W. Wolff (Neukirchen-Vluyn, I981), pp. I I-29.

${ }^{78}$ J.-L. Ska, "L'appel d'Abraham et l'acte de naissance d'Israël. Genèse i2,1-4a," dans M. Vervenne et J. Lust (éd.), Deuteronomy and Deuteronomic Literarture. Festschrift C.H.W. Brekelmans, BETL I33 (Leuven, I997), pp. 367-389. 
intègre l'histoire des origines dite "yahwiste" (qu'il date du début de l'ère postexilique) dans le récit sacerdotal. ${ }^{79}$

D'une certaine manière, cette indépendance des textes non-P dans le cycle des origines se trouve mise en question par un courant de la recherche actuelle qui considère ces textes comme des suppléments à $\mathrm{P}$, renversant ainsi l'un des piliers traditionnels de l'exégèse de Gn I-II. ${ }^{80}$ Pourtant, l'étude de Witte montre qu'il reste possible d'imaginer un récit non sacerdotal indépendant. Les parallèles qui existent entre $\mathrm{Gn}$ 2-8* et les mythes d'origines mésopotamiens (cf. notamment Atrahasis) rendent cette hypothèse assez plausible. ${ }^{81}$ La possibilité d'une combinaison de l'histoire des origines avec celle des Patriarches au niveau présacerdotal déjà dépend alors en grande partie du statut de Gn I2,I-3. Il faudrait également approfondir la question de savoir si le lien entre le cycle des origines et l'histoire patriarcale s'est opéré à un stade où la Genèse était encore indépendante de ce qui suit ${ }^{82}$ ou si le rédacteur de Gn 2-8 avait déjà en vue le Pentateuque, voire un ensemble littéraire encore plus grand.

\subsubsection{Une ancienne histoire de Moïse ou de la sortie d'Egypte?}

L'indépendance primitive d'Ex I-I5 a souvent été soulignée. Johannes Pedersen y voyait une ancienne légende de la Pâque. ${ }^{83}$ Parmi les auteurs récents, Peter Weimar, Zenger et Ernst Axel Knauf ont postulé l'existence d'une histoire prémonarchique de la sortie d'Egypte, alors

${ }^{79} \mathrm{M}$. Witte, Die biblische Urgeschichte. Redaktions- und theologiegeschichtliche Beobachtungen zu Genesis I,I-II,26, BZAW 265 (Berlin, New York, I998), pp. I99-20I.

${ }^{80}$ Gf. Blenkinsopp, Pentateuch, pp. 54ss.; J.-L. Ska, "El Relato del Diluvio. Un relato sacerdotal y algunos fragmentos redaccionales posteriores," Estudios Biblicos 52 (I994), pp. 37-62; E. Otto, "Die Paradieserzählung Genesis 2-3: Eine nachpriesterliche Lehrerzählung in ihrem religionshistorischen Kontext," dans A.A. Diesel et al. (éd.), "Fedes Ding hat seine Zeit...". Studien zur israelitischen und altorientalischen Weisheit. Diethelm Michel zum 65. Geburtstag, BZAW 24I (Berlin, New York, 1996), pp. I67-192; T. Krüger, "Das menschliche Herz und die Weisung Gottes. Elemente einer Diskussion über die Möglichkeiten und Grenzen der Tora-Rezeption im Alten Testament," dans T. Krüger et R.G. Kratz (éd.), Rezeption und Auslegung im Alten Testament und in seinem Umfeld. Ein Symposium zum Anlass des 6o. Geburtstags von Odil Hannes Steck, OBO ${ }_{153}$ (Freiburg CH, Göttingen, 1997), pp. 65-92.

${ }^{81}$ Cf. en ce sens Knauf, "Geschichte," p. Io8. Ceci n'exclut nullement la possibilité que certains textes traditionnellement considérés comme "J" soient plutôt à attribuer au travail d'un rédacteur, cf. Witte, Urgeschichte, passim.

${ }^{82}$ Rappelons que la rédaction des toledot se limite en fait au livre de la Genèse (Ex 6,16. Ig et $\mathrm{Nb}_{3}$, I ont une construction et une fonction différentes).

${ }^{83}$ J. Pedersen, "Passahfest und Passahlegende," ZAW 52 (I934), pp. I6 I-I75. 
qu'Albertz identifie en $\mathrm{Ex}$ I-I5 les restes d'une ancienne histoire de Moïse (de l'époque de Jéroboam I), ainsi qu'une histoire des plaies et de la sortie d'Egypte datant de l'époque exilique. ${ }^{84}$ Wolfgang Oswald souligne également l'indépendance primitive d'Ex I-I5, qui aurait été transformé à l'époque exilique en une "Exodus-Gottesberg-Erzählung" (allant d'Ex I-24*). ${ }^{85}$ Tout récemment, Jan Christian Gertz a reconstruit en détail les contours d'un récit de l'exode indépendant tout en restant assez discret sur le Sitz im Leben de celui-ci. ${ }^{86}$

Reste pourtant la question de la plausibilité d'une telle reconstruction. Certes, l'existence d'une tradition autonome de la sortie d'Egypte n'est pas à exclure. Il faut néanmoins s'interroger sur l'aboutissement de cette tradition. La sortie d'Egypte ne doit-elle pas déboucher sur l'arrivée du peuple dans le pays, ou au moins à la montagne de Dieu? Les hypothèses concernant l'existence d'une vita Mosis présupposant la chute du Royaume du Nord (Blum) ou d'une histoire de Moïse antiassyrienne du VIIème siècle (Otto) méritent considération. ${ }^{87}$ Toutefois, les contours d'une telle œuvre demeurent assez flous. Pour Otto, son point culminant se trouve dans les prescriptions cultuelles et la conclusion d'alliance en Ex $34^{*}$. Une telle histoire a-t-elle déjà contenu des récits concernant le séjour dans le désert? La réponse n'est pas aisée. Les quelques mentions du désert dans le livre d'Osée (Os 2,5; 9, IO; 13,5) ne présupposent nullement l'existence d'une ancienne tradition du désert, qui serait préservée dans le Pentateuque. ${ }^{88}$ Les histoires de révolte dans le livre des Nombres semblent plutôt présupposer les textes et la théologie de l'histoire dtr.

${ }^{84}$ P. Weimar, E. Zenger, Exodus: Geschichten und Geschichte der Befreiung Israels, SBS 75 (Stuttgart, I975); E.A. Knauf, Midian. Untersuchungen zur Geschichte Palästinas und Nordarabiens am Ende des 2. Jahrtausends v.Chr., ADPV (Wiesbaden, 1988), pp. I3 ${ }^{1-135}$; R. Albertz, Religionsgeschichte Israels in alttestamentlicher Zeit, GAT 8 (Göttingen, I992), pp. 71-72 et p. 727 .

${ }^{85}$ W. Oswald, Israel am Gottesberg. Eine Untersuchung zur Literaturgeschichte der vorderen Sinaiperikope Ex 19-24 und deren historischem Hintergrund, OBO 159 (Fribourg CH, Göttingen, 1998), pp. I21-I25.

${ }_{86}$ Gertz, Exoduserzählung, cf. le résumé aux pp. 394-396. Le récit primitif aurait contenu l'oppression d'Israël, la naissance, la fuite et la vocation de Moïse (Ex I-4*), ainsi que le récit de la sortie $\left(\mathrm{Ex} \mathrm{I}_{3}-\mathrm{I} 4^{*}\right)$.

87 Blum, Studien, pp. 216-218; Otto, "Mose und das Gesetz".

${ }^{88}$ Cf. T.B. Dozeman, "Hosea and the Wilderness Wandering Tradition," dans S.L. Mckenzie et T. Römer (éd.), Rethinking the Foundations. Historiography in the Ancient World and in the Bible. Essays in Honour of John Van Seters, BZAW 294 (Berlin, New York, 2000), pp. 55-70. 
Dans le cadre de cette problématique, il faudrait bien sûr également reprendre le dossier de la péricope du Sinaï. Bien qu'il existe actuellement un certain consensus sur le fait qu'Ex Igss. est une composition récente qui transforme la tradition de Sion, l'origine et la fonction de ces textes sont loin d'être clairs.

\subsubsection{Les codes législatifs}

Une question parallèle qui se pose concerne l'intégration de la "loi" dans la théophanie du Sinaï. L'indépendance originelle et la succession chronologique des collections suivantes: code d'alliance, code deutéronomique et code de sainteté est acceptée par la majorité des chercheurs, ${ }^{89}$ bien que Van Seters ait mis en question la priorité du code d'alliance sur le code deutéronomique..$^{90}$ Si l'on admet néanmoins une origine du code d'alliance aux alentours du VIIIème siècle, ${ }^{91}$ il faut cependant encore résoudre la question de sa fonction et de son insertion en Ex I9ss.*. Contrairement à Blum, qui situe l'intégration du code d'alliance dans la péricope du Sinaï à un stade pré-dtr déjà ${ }^{92}$ plusieurs travaux récents attribuent cette insertion à une rédaction postsacerdotale (cf. notamment Crüsemann, Levin, Otto) ${ }^{93}$ Cette proposition vise à tenir compte de l'autonomie relative du code d'alliance dans son contexte narratif. Elle pose toutefois un problème, puisqu'on comprend mal un retour du code d'alliance durant l'époque perse, surtout si l'on adopte l'idée que le code deutéronomique a été rédigé pour remplacer le code d'alliance. En ce sens, la thèse d'Oswald paraît plus séduisante, puisqu'il considère l'édition du code d'alliance comme une sorte de "constitution" à l'époque de l'exil pour les Judéens restés dans le pays et gouvernés par Guedalias. ${ }^{94}$

${ }^{89}$ Cf. Ska, Introduction, pp. 63-8o; et pour la relation entre Ex $20-23^{*}$ et Dt $12-26^{*}$, cf. B.M. Levinson, Deuteronomy and the Hermeneutics of Legal Innovation (New York, Oxford, 1997).

${ }_{90}$ Cf. notamment J. Van Seters, "The Law of the Hebrew Slave," ZAW 108 (1996), pp. $534-546$.

91 L. Schwienhorst-Schönberger, Das Bundesbuch (Ex 20,22-23,33). Studien zu seiner Entstehung und Theologie, BZAW I88 (Berlin, New York, I99o); Y. Osumi, Die Kompositionsgeschichte des Bundesbuches Exodus 20,22b-23,33, OBO I05 (Freiburg CH, Göttingen, 199I); F. Crüsemann, Die Tora. Theologie und Sozialgeschichte des alttestamentlichen Gesetzes (München, 1992), pp. I32-234.

92 Studien, p. 94 .

${ }^{93}$ Crüsemann, Tora, p. 23o; Levin, Fahwist, pp. 365-366; E. Otto, Theologische Ethik des Alten Testaments, ThW 3.2 (Stuttgart-Berlin-Köln, I994), pp. 67-I I .

94 Israel, p. ${ }^{2} 3^{8 .}$ 
La question des rapports entre les codes législatifs et les couches rédactionnelles constitue également un point important dans le cadre de la discussion actuelle. Pour Dt 12-26*, dont l'origine peut aisément être située au VIIème siècle, le lien avec la rédaction dtr des Prophètes antérieurs est évident. De même, l'intégration du Code de sainteté dans un travail rédactionnel de type sacerdotal semble fort plausible. Pour le code d'alliance, la question semble plus complexe.

D'une manière générale, la coexistence de ces trois collections à l'intérieur de la Torah ne devrait pas seulement inspirer des réflexions de type théologique, mais également inviter à un affinement des hypothèses rédactionnelles.

\subsection{Unités autonomes et rédacteurs englobants}

La tendance actuelle que nous venons brièvement de discuter, et qui insiste à nouveau sur l'autonomie relative de certains grands ensembles rejoint, d'une certaine façon, une direction de la recherche déjà esquissée par Rendtorff dans son travail sur les "unités majeures" dans le Pentateuque ${ }^{95}$ Rendtorff, qui avait repris l'approche de l'Überlieferungsgeschichte de Noth, y avait néanmoins introduit un changement de perspective. Alors que pour Noth, la mise en commun des différents "thèmes" du Pentateuque s'était encore opérée à l'époque prémonarchique, Rendtorff attribuait celle-ci à une rédaction de type deutéronomique. Les modèles de la composition D, ou encore du Yahwiste post-dtr, rejoignent cette idée. Néanmoins, tout en reconnaissant une certaine indépendance aux matériaux intégrés par $\mathrm{J}$ ou $\mathrm{D}$, ces modèles globaux avaient tendance à les délaisser pour se préoccuper presque exclusivement du travail des rédacteurs. ${ }^{96}$ Le retour partiel à une théorie des fragments peut ainsi être compris comme une réaction à l'encontre d'un modèle du Pentateuque sans doute trop simpliste. Il reste néanmoins à se demander si, outre les codes législatifs, les unités majeures en $\mathrm{Ex}-\mathrm{Nb}$ peuvent être aussi précisément reconstruites que pour la Genèse (cf. notamment les travaux de de Pury et de Blum sur le cycle de $\left.\mathrm{Jacob}^{97}\right)$. Cette différence est peut-être due au fait que l'histoire rédac-

\footnotetext{
${ }_{95}^{5}$ Das überlieferungsgeschichtliche Problem.

${ }^{96}$ Cette tendance est poussé à l'extrême chez R.N. Whybray, Introduction to the Pentateuch (Grand Rapids MI, I995), lequel postule un seul auteur derrière le Pentateuque tout en reconnaissant que celui-ci s'est servi de traditions diverses, qui ne peuvent toutefois plus être reconstruites.

97 A. de Pury, "Le cycle de Jacob comme légende autonome des origines d'Israël,"
} 
tionnelle du Pentateuque se présente de manière fort différente pour le livre de la Genèse d'un côté, et pour les livres suivants de l'autre. La séparation la plus évidente à l'intérieur du Pentateuque est sans aucun doute celle qui existe entre la Genèse et Ex-Dt, et elle apparaît jusque dans les conclusions de chacun de ces livres. ${ }^{98}$

Ces observations signifient, à mon sens, qu'il faudrait peut-être envisager une meilleure différenciation des modèles explicatifs à l'intérieur de la Torah.

\section{Quelques considérations et suggestions méthodologiques}

\section{I. La fin des théories globales?}

Il est notoire que la théorie documentaire s'est développée à partir des travaux sur le livre de la Genèse. Cette théorie était encore applicable à la première partie du livre de l'Exode, mais déjà il s'avérait difficile d'expliquer la péricope du Sinaï à l'aide de ce modèle. En ce qui concerne le livre des Nombres, Noth lui-même faisait remarquer: "Si l'on partait du livre des Nombres, on n'aurait guère l'idée de sources parallèles, mais on penserait plutôt à une mise en commun peu systématique de nombreux morceaux, datant d'époques diverses et ayant des contenus et des caractéristiques fort différents les uns

dans J.A. Emerton (éd.), Congress Volume Leuven 1989, VT.S 43 (Leiden, I99I), pp. 78-96; "Situer le cycle de Jacob" dans A. Wénin (éd.), Studies in the Book of Genesis, BETL 155 (Leuven, 200o), pp. 123-143. E. Blum, Die Komposition der Vätergeschichte, WMANT 57 (Neukirchen-Vluyn, I984). Pour une datation différente et une critique d'une certaine conception de la tradition orale, cf. H.M. Wahl, Die Jakobserzählungen. Studien zu ihrer mündlichen Überlieferung, Verschriftung und Historizität, BZAW $25^{8}$ (Berlin, New York, 1997). Il est possible que le cycle d'Abraham ait d'abord été conçu indépendamment du cycle de Jacob, mais néanmoins plus tard que celui-ci, cf. par ex. I. Fischer, Die Erzeltern Israels. Feministisch-theologische Studien zu Genesis I2-36, BZAW 222 (Berlin, New York, I994). Il n'est guère possible d'envisager un cycle d'Abraham avant l'exil (cf. par contre A. Lemaire, "Vues nouvelles sur la tradition biblique d'Abraham," dans A. Lemaire [éd.], Les routes du Proche-Orient. Des séjours d'Abraham aux caravanes de l'encens [Paris, 200o], pp. 21-31 et I17-121, qui postule la première mise par écrit aux alentours de l'an Iooo, à l'époque de David).

${ }^{98}$ E. Ben Zvi, "The Closing Words of the Pentateuchal Books: A Clue for the Historical Status of the Book of Genesis within the Pentateuch," BN 62 (1992), pp. 7-1o, a montré que la Genèse se différencie dans sa conclusion des livres d'Ex, Lv, Nb et Dt, dont les derniers versets sont construits comme des renvois mutuels. L'unité d'Ex-Dt est déjà soulignée par le fait que ces livres coïncident avec l'histoire de Moïse (de sa naissance, en Ex 2, jusqu'à sa mort, en Dt 34). 
des autres (hypothèse des fragments)" ${ }^{99}$ Cette observation mérite toute notre attention. La recherche sur le Pentateuque s'est en effet peu occupée de la question de l'apparition et de la constitution des cinq rouleaux. Si l'on observe les différents livres de la Torah, ${ }^{100}$ on constate que $\mathrm{Gn}, \mathrm{Ex}, \mathrm{Lv}$ et Dt possèdent chacun une structure assez claire et cohérente. ${ }^{101}$ Ceci est beaucoup moins vrai pour le livre des Nombres, comme le montre la discussion sans fin concernant le plan du livre. ${ }^{102}$ Ce manque apparent de cohérence pourrait s'expliquer par l'idée que le livre des Nombres a été le dernier ensemble du Pentateuque à prendre forme, et qu'on y a intégré des éléments qui ne pouvaient déjà plus trouver place dans les autres livres. ${ }^{103}$ On pourrait alors imaginer pour $\mathrm{Nb}$ un processus de Fortschreibung semblable à celui que Walter Zimmerli a suggéré pour le livre d'Ezéchiel. ${ }^{104}$

\subsection{Fortschreibung dans le livre des Nombres}

Pour Noth, les derniers chapitres de $\mathrm{Nb}$ se sont constitués à la suite de multiples ajouts d'origines diverses, qui furent insérés au cours de la dernière phase de l'édition du Pentateuque. ${ }^{105}$ Peut-être faut-il généraliser cette hypothèse, et l'élargir à une grande partie du livre. Pour ne donner qu'un exemple, les chapitres II-I2 peuvent être compris comme le résultat de réinterprétations successives, qui annoncent déjà un certain type d'herméneutique rabbinique. ${ }^{106}$

99 M. Noth, Das 4. Buch Mose. Numeri, ATD 7 (Göttingen, ${ }^{3}$ I977): "Nimmt man das 4. Mosebuch für sich, so käme man nicht leicht auf den Gedanken an 'durchlaufende Quellen', sondern eher auf den Gedanken an eine unsystematische Zusammenstellung von zahllosen Überlieferungsstücken sehr verschiedenen Inhalts, Alters und Charakters ('Fragmentenhypothese')" (p. 8).

100 Pour la question des titres et des conclusions, cf. H.-P. Mathys, "Bücheranfänge und -schlüsse," dans Vom Anfang und vom Ende: fün alttestamentliche Studien, BEATAJ 47 (Frankfurt/M. et al., 2000), pp. I-29.

$101 \mathrm{Gn}$ : origines du monde et Patriarches; Ex: sortie d'Egypte, don de la loi et fondation du sanctuaire au Sinaï; Lv: prescriptions cultuelles et éthiques; Dt: testament de Moïse.

102 Cf. en dernier lieu Artus, Etudes sur le liore des Nombres, pp. 15-40.

103 Ceci expliquerait par exemple le prolongement du séjour au Sinaï jusqu'en $\mathrm{Nb}$ Io, qui permet ainsi par exemple de préciser certains règlements concernant la Pâque ( $\mathrm{Nb}$ 9), alors même que ces précisions trouveraient pourtant une place plus adéquate après $\mathrm{Ex} 12$ ou Lv 23.

${ }^{104}$ W. Zimmerli, Ezechiel, BK XIII/1 + 2 (Neukirchen-Vluyn, ${ }^{2}$ I979).

105 Numeri, pp. II-I2.

106 Cf. pour la suite T.C. Römer, "Nombres I I-12 et la question d'une rédaction deutéronomique dans le Pentateuque," dans M. Vervenne et J. Lust (éd.), Deuteronomy and 
L'histoire des cailles et du don de l'esprit en $\mathrm{Nb}$ I r relève apparemment d'une rédaction de type prophétique (cf. notamment les parallèles entre $\mathrm{Nb}$ II,I7 et $\mathrm{Ez} 37,5 ; \mathrm{Nb}$ II,2I et $\mathrm{Ez}$ 37,I.3; $\mathrm{Nb}$ II,23 et Es 50,$2 ; 59, \mathrm{I} ; \mathrm{Nb}$ I I,29 et Es 44,3; Ez 36,27, etc.) qui présuppose à la fois "D/J" et "P" (cf. par ex. la reprise d'Ex 24,9-I en Nb II,I7.25), et qui édite un récit antérieur, lequel constitue peut-être déjà un midrash d'Ex i6. Cette rédaction réinterprète ce récit à partir d'Ex 18 et de $\mathrm{Dt}$ I,gss. Bien que, dans l'ensemble, il ait été tenu à l'écart de la Torah, le courant prophético-eschatologique a néanmoins pu s'intégrer dans le livre des $\mathrm{Nb}$, en cherchant à légitimer une conception moins institutionnelle et plus "démocratique" de la prophétie par le biais de la figure de Moïse lui-même. ${ }^{107}$ Plus tard, cette rédaction prophétique a été corrigée au chapitre 12 par une "rédaction mosaïque" (I2,2-9). Cette rédaction est proche de l'idéologie dtr tardive, voire de la "rédaction du Pentateuque" (cf. Nb I2,6-8 et Dt 34,10-12). Elle insiste sur l'incompatibilité de Moïse et prône une distinction entre celui-ci et les courants prophétiques. Finalement, un autre rédacteur, peut-être issu de la diaspora, a greffé autour de ce récit de contestation un autre récit

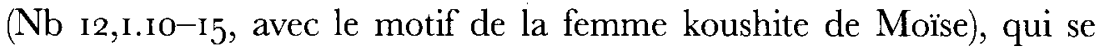
réclame de l'autorité de Moïse pour prôner une conception ouverte du judaïsme, et qui refuse notamment l'idéologie d'Esdras-Néhémie et l'interdiction des "mariages mixtes". Il serait tentant de voir si ces mêmes rédactions se retrouvent ailleurs. Mais il est tout aussi possible que nous ayons affaire ici à un processus de Fortschreibung face auquel la quête de couches rédactionnelles parcourant l'ensemble du livre des Nombres, voire du Pentateuque, devient aléatoire.

\subsection{Pour une prise en compte de la diversité rédactionnelle des livres du Pentateuque}

La situation actuelle des recherches sur le Pentateuque nous invite à être attentifs aux spécificités de certains ensembles de la Torah. Ainsi par exemple, Gertz a émis l'idée que le statut de $\mathrm{P}$ pourrait être différent d'un livre à un autre. Pour lui, contrairement à ce qui est le cas

Deuteronomic Literature. Festschrift C.H.W. Brekelmans, BETL I33 (Leuven, I997), pp. 481498.

${ }_{107}$ Les versets $11,5.18 \mathrm{~b}-20$ introduisant le motif de la nostalgie d'Egypte (cf. Ex I3,17b; 14,II-12; I6,3; I7,3; $\mathrm{Nb} 14,2-4 ; 16,12-14 ; 20,4-5 ; 21,4)$ appartiennent peutêtre encore à une autre rédaction, qui veut renforcer le lien entre Ex et $\mathrm{Nb}$. Otto, Deuteronomium, p. 108, n. 404, envisage la "Hexateuchredaktion". 
en Gn I-I I et en Ex I-I4, P s'explique mieux en Gn i2ss. comme une rédaction que comme un texte véritablement autonome. $\mathrm{P}$, l'artisan du pont littéraire entre Genèse et Exode, aurait dès le début intégré dans sa présentation des Patriarches au moins une partie du matériel non sacerdotal. ${ }^{108}$ Une telle proposition, qui doit encore être approfondie, a le mérite de prendre en compte les particularités de certains grands ensembles et de déplacer quelque peu le débat sur $\mathrm{P}$.

L'autre question à reprendre est celle du profil spécifique des différents livres du Pentateuque. Le livre de l'Exode fait apparaître un mélange de style sacerdotal et dtr, contrairement à la Genèse où la présence de textes dtr se fait beaucoup plus discrète. ${ }^{109}$ Le livre du Lévitique est un produit entièrement sacerdotal, ${ }^{110}$ tandis que le Deutéronome ne connaît guère d'interventions de type sacerdotal. Reste alors le livre des Nombres, lequel a pu accueillir des textes tardifs qui ne trouvaient déjà plus de place ailleurs, et dont certains émanent peutêtre d'autres milieux que ceux qui avaient déjà clos, d'une certaine manière, le "Tritoteuque" (Gn-Lv*) et l'historiographie dtr (Dt-2Rois). $\mathrm{Si}$ les recherches récentes qui trouvent la conclusion originale de $\mathrm{P}$ dans la péricope du Sinaï ont raison, on pourrait alors envisager que le livre des Nombres ait notamment pris forme lors de la mise en commun, d'une part d'un ensemble Gn-Lv édité sous égide sacerdotale, et d'autre part du Dt, détaché de l'histoire dtr. Le livre des Nombres deviendrait ainsi le berceau des rédactions tardives mêlant le style sacerdotal au style dtr, rédactions qui parcourent avec plus ou moins d'intensité l'ensemble du Pentateuque, voire de l'Hexateuque. L'existence de telles rédactions est en effet de plus en plus admise. ${ }^{11}$ Leur caractère précis reste toutefois à définir.

108 Exoduserzählung, p. 39I, cf. également p. 388 et la note I7r: "In der Väter- und Josephsgeschichte stößt die Rekonstruktion einer selbständigen Quellenschrift $\mathrm{P}$ bekanntlich an ihre Grenzen. Daß P in diesem Bereich anders als in der Exoduserzählung nicht als strukturierende Grundlage gedient hat ist, wie mir scheint, evident". Pour une vision différente cf. A. de Pury, "Abraham: The Priestly Writer's 'Ecumenical' Ancestor," dans S.L. McKenzie et T. Römer (éd.), Rethinking the Foundations: Historiography in the Ancient World and in the Bible. Essays in Honour of John Van Seters, BZAW 294 (Berlin, New York, 200o), pp. i63-18i.

${ }^{109}$ Cf. Levin, Fahwist, p. 436 et H. Seebass, Genesis I. Urgeschichte $(I, I-I I, 26)$ (Neukirchen-Vluyn, I996), p. 34 .

${ }^{110}$ A l'exception de quelques interventions rédactionnelles tardives et deutéronomisantes.

111 Cf. notamment les travaux d'Otto sur la Hexateuchredaktion et la Pentateuchredaktion, ainsi que Levin, Jahwist; N. Lohfink, "Gab es eine deuteronomische Bewegung?," dans 


\section{En guise de conclusion: \\ un compromis possible pour un document de compromis?}

Comme nous l'avons souligné en ouverture, le débat sur la formation de la Torah se caractérise aujourd'hui principalement par la coexistence de théories fort différentes, voire ouvertement contradictoires, et il serait certes démagogique de chercher à dresser un tableau réconciliant toutes les approches contemporaines de l'exégèse historicocritique du Pentateuque. L'éclatement de l'exégèse actuelle du Pentateuque correspond en quelque sorte à l'éclatement des discours dans une société dite "postmoderne". Ceci ne signifie pourtant pas que l'exégèse historico-critique s'est désavouée, comme certains aimeraient tant le faire croire. L'exégèse, comme tout discours raisonné, ne peut se faire qu'en contact et en réaction avec la société dans laquelle elle s'élabore. Il n'est d'ailleurs peut-être pas inutile de rappeler que de nombreux résultats des débuts des recherches diachroniques restent toujours valables; et ce n'est sans doute pas un hasard si l'on observe aujourd'hui à divers niveaux un retour vers le maître Wellhausen, soit dans le cadre d'une version modifiée de l'hypothèse documentaire, qui renonce au Yahwiste salomonien (Seebass), soit dans le cadre d'une Literarkritik qui s'inspire étroitement du paradigme wellhausenien, tout en le modifiant (Levin, Kratz). L'insistance nouvelle sur une sorte de théorie des fragments reprend le programme de Noth, et postule à sa manière l'existence d'ensembles littéraires à l'époque monarchique. On notera que l'intérêt que porte cette théorie des fragments reformulée aux différences idéologiques entre certaines traditions (notamment dans l'opposition des traditions sur les Patriarches aux traditions sur Moïse) pourrait rejoindre une idée assez répandue aujourd'hui, celle du Pentateuque comme document de compromis. Il existe de nos jours un quasi-consensus sur le fait que c'est l'époque perse qui fournit la vraie date de naissance de la Torah. La publication du Pentateuque, ou en tout cas d'un Proto-Pentateuque, se comprend comme une tentative du judaïsme naissant pour définir sa nouvelle identité face aux changements politiques, économiques et religieux auxquels il se trouve confronté. Dans cette perspective, on avait pensé pouvoir mettre en

W. Groß (éd.), Feremia und die "deuteronomistische Bewegung”, BBB 98 (Weinheim, I995), pp. 313-382; H.-C. Schmitt, "Die Suche nach der Identität des Jahweglaubens im nachexilischen Israel," dans J. Mehlhausen (éd.), Pluralismus und Identität, VWGTh 8 (Gütersloh, 1995), pp. 259-278, parmi bien d'autres. 
rapport la publication de la Torah avec la supposée institution perse de l'autorisation impériale. ${ }^{112}$ En réalité, il n'est pas si évident qu'une telle autorisation ait vraiment existé; les textes cités en faveur de cette hypothèse ne sont d'ailleurs guère compatibles avec le Pentateuque, et il est de toute façon douteux que les Perses aient pris eux-mêmes l'initiative de donner un statut officiel à des traditions locales. ${ }^{113}$ Ceci dit, un texte comme Esd 7 cherche visiblement à faire bénéficier la loi d'Esdras de la légitimité de l'autorité achéménide, et on peut imaginer en ce sens que les protagonistes de la Torah aient sollicité un certain soutien auprès du pouvoir perse pour faire reconnaitre la nouvelle loi en Judée et en Samarie. Le rassemblement de différents codes législatifs et de traditions narratives très diverses fait indubitablement du Pentateuque une littérature de compromis. ${ }^{114}$ Mais c'est là un compromis qui ne se limite pas simplement à la cohabitation côte à côte du courant sacerdotal $(\mathrm{P})$ et du courant "laïc" (D ou J), ${ }^{115}$ et qui ne veut pas gommer les divergences entre leurs options théologiques. Au contraire, pour qui sait écouter et lire, celles-ci sont évidentes. La cohérence du Pentateuque, c'est sa diversité. Et l'exégèse historico-critique, malgré ses résultats toujours provisoires et qui doivent être continuellement revus, participe à cette quête visant à mieux comprendre une unité qui n'implique pourtant aucune uniformité.

112 L'auteur de cette théorie est P. Frei, "Zentralgewalt und Lokalautonomie im Achämenidenreich," dans P. Frei et K. Koch, Reichsidee und Reichsorganisation im Perserreich, OBO 55 (Freiburg CH, Göttingen, I996), pp. 5-I3I. Cette théorie jouit d'une grande popularité, cf. parmi d'autres F. Crüsemann, "Le Pentateuque, une Tora. Prolégonıènes à l'interprétation de sa forme finale," dans A. de Pury (éd.), Le Pentateuque en question, Le Monde de la Bible I9 (Genève, ${ }^{2}$ I99I), pp. 339-36o; Blum, Studien, pp. 345-360; R. Albertz, Religionsgeschichte, pp. 495-535; E.A. Knauf, "Audiatur et altera pars. Zur Logik der Pentateuchredaktion," BiKi 53 (1998), pp. I 18-126.

${ }^{113}$ Cf. les remarques critiques de U. Rüterswörden, "Die persische Reichsautorisation der Thora: Fact or Fiction," ZAR I (I995), pp. 47-6i; J. Wiesehöfer, "'Reichsgesetz' oder 'Einzelfallgerechtigkeit'. Bemerkungen zu P. Freis These von der achaimenidischen 'Reichsautorisation'," ZAR I (1995), pp. 36-46; ainsi que Ska, Introduction, pp. 310-32 I.

114 Cf. notamment B.J. Diebner, "Zur hermeneutischen Bedeutung der Spannung zwischen Judäa und Samarien für das Verständnis des TNK als Literatur," dans M. Prudky (éd.), Landgabe. Festschrift für Jan Heller (Praha-Kampen, 1995), pp. 86-132; Knauf, "Audiatur".

115 Le roman de Joseph, par exemple, me semble défendre les intérêts d'un judaïsme libéral de la diaspora. 